\title{
ON THE DIMENSIONS OF CERTAIN SPACES OF HOMEOMORPHISMS $\left({ }^{1}\right)$
}

\author{
BY \\ BEVERLY L. BRECHNER( $(2)$
}

\section{Preliminaries.}

INTRODUCTION. This paper studies the dimensions of the groups of all homeomorphisms of certain metric continua. It is divided into the following (overlapping) parts:

§2: Continua with nonzero dimensional groups of homeomorphisms,

§3: Applications of Theorem 2.1,

$\S 4$ : 1-dimensional continua,

$\S 5$ : Continua with finite, positive dimensional groups of homeomorphisms.

In $\S 2$, we establish sufficient conditions for the group of all homeomorphisms of a continuum to be nonzero dimensional, and thus obtain a large class of continua with this property - the (to be defined) locally setwise homogeneous continua.

It is well known and easy to show (see Theorem 1.1) that the group of homeomorphisms of an arc is $\infty$-dimensional. The component of the identity in this case is large. However it is not hard to show that the groups of all homeomorphisms of the universal plane curve and universal curve are totally disconnected. It therefore seems likely that they are zero-dimensional. In fact, such a result was announced (erroneously) by R. D. Anderson [1], who called it to my attention and suggested the general problem of this paper to me. His argument was for total disconnectivity instead of 0 -dimensionality and is given in Theorem 1.2. However, corollaries of our main theorem of $\$ 2$ show that each of these groups is at least 1-dimensional.

In $\$ 3$ the above corollaries are obtained, as well as an interesting corollary of the proof of Theorem 2.1. This corollary asserts that the group of those homeomorphisms of $S^{n}, n>1$, which carry a fixed, countable, dense subset of $S^{n}$ onto itself, is at least 1-dimensional.

In $\S 4$, we investigate the 1-dimensional continua. Our main result asserts that

Presented to the Society, November 21, 1964; received by the editors November 23, 1964.

(1) This is essentially the author's doctoral dissertation at Louisiana State University in Baton Rouge, August 1964.

(2) The author would like to thank her adviser, Professor R. D. Anderson, for his help. She would also like to thank the referee for many helpful comments and criticisms. 
regular curves may have zero or infinite dimensional groups of homeomorphisms, and only these, while rational curves may have finite, positive dimensional groups.

Finally in $\S 5$, we construct, for each positive integer $n$, examples of continua $M_{n}$ whose full groups of homeomorphisms are totally disconnected, abelian, and homeomorphic to the product of $n$ 1-dimensional groups. Thus $1 \leqq \operatorname{dim} G\left(M_{n}\right) \leqq n$. In particular, for $n=1, G\left(M_{n}\right)$ is exactly 1-dimensional. The continua $M_{n}$ may be constructed as rational curves.

The above results suggest many other questions, which are raised, but unanswered, in this paper.

Definitions AND Conventions. All spaces are separable metric.

If $X$ is a compact, metric space, $G(X)$ (or $G$ when no confusion arises) will denote the group of all homeomorphisms of $X . H$ or $H(X)$ will denote a subgroup of $G$.

The metric on $X$ will be denoted by $d$, and $d(x, y)$ denotes the distance between $x$ and $y$.

The metric in $G(X)$ will also be denoted by $d$. We define the metric in $G(X)$ as follows $\left({ }^{3}\right)$ :

$$
\text { for } g, h \in G(X), \quad d(g, h)=\operatorname{lub}_{x \in X}\{d(g(x), h(x))\} .
$$

If $\mathscr{U}$ is a collection of sets $\mathscr{U}^{*}$ denotes the union of the elements of $\mathscr{U}$. If $\mathscr{V}$ is also a collection of sets, $\mathscr{V}$ is said to refine $\mathscr{U}$ if each element of $\mathscr{V}$ is a subset of some element of $\mathscr{U} . \mathscr{V}$ is called a closed refinement of $\mathscr{U}$ if the closure of each element of $\mathscr{V}$ is a subset of some element of $\mathscr{U}$. Mesh of $\mathscr{U}$ means the least upper bound of the diameters of the elements of $\mathscr{U}$.

$C(U)$ denotes the complement of the set $U$.

If $g$ is a homeomorphism, $g$ is supported on $U$ means $g$ is the identity on $C(U)$.

In $f: X \longrightarrow X$ the double headed arrow means $f$ is a function from $X$ onto $X$.

$X^{0}$ denotes the interior of $X$.

The dimension of a set $X$ is denoted by " $\operatorname{dim} X$ ". We use inductive dimension (for separable metric spaces) defined as in [6].

Preliminary Theorems.

THEOREM 1.1 (WELL-KNOWN). The group of all homeomorphisms of a compact n-manifold, $M$, is infinite dimensional.

Proof. Let $\left\{C_{i}\right\}_{i=1}^{\infty}$ be a sequence of disjoint $n$ cells of $M$, converging to a point of $M$. For each $i, 1 \leqq i<\infty$, let $\sigma_{i}$ be an arc of homeomorphisms of $M$ onto itself, supported on $C_{i}$, and denoted by $\left\{h_{i, \alpha}: 0 \leqq \alpha \leqq 1 / i\right\}$. Let $H=\{h \in G(M): h$ is supported on $\bigcup_{i=1}^{\infty} C_{i}$, and, for each $i$, there is an $\alpha_{i}$ such that $\left.h\left|C_{i}=h_{i, \alpha_{i}}\right| C_{i}\right\}$.

(3) The following is also a metric for $G(X): \rho(g, h)=d(g, h)+d\left(g^{-1}, h^{-1}\right)$, where $d$ denotes the metric defined above. Then $\rho$ is a complete metric for $G(X)$ and is equivalent to $d$. However, we prefer to use the metric $d$, since it is intuitively easier to understand.

It is well known that $G(X)$ is separable. 
Then $H$ is homeomorphic to a Hilbert cube, since each element $h \in H$ can be identified with an infinite sequence $\left(h_{1, \alpha_{1}}, h_{2, \alpha_{2}}, \cdots, h_{n, \alpha n}, \cdots\right)$ where $\alpha_{n} \in[0,1 / n]$. Therefore $H$, and thus $G(M)$, is infinite dimensional.

Theorem 1.2 (R. D. Anderson). The group of homeomorphisms, G, of the universal plane curve, $M$, is totally disconnected.

Proof $\left({ }^{4}\right)$. We will show that any pair of elements of $G$ can be separated; that is, given $g, h \in G$, there exist two mutually separate sets $A$ and $B$ such that $g \in A$, $h \in B$, and $A \cup B=G$. We first note that $M$ can be thought of as a circle $C_{0}$ plus its interior in $E^{2}$, with the interiors of a countable dense set of pairwise disjoint circles, $\left\{C_{i}\right\}_{i=1}^{\infty}$, removed, where each $C_{i}$ is in the interior of $C_{0}$, diam $C_{i} \rightarrow 0$, and no $C_{i}$ is a subset of any $C_{j}^{0}$. This follows from the main result of [3].

Let $g_{0}, h_{0} \in G, g_{0} \neq h_{0}$. We shall show that there exists an $n_{0}$ such that $h_{0}\left(C_{n_{0}}\right) \cap g_{0}\left(C_{n_{0}}\right)=\phi$. There exists a point $x$ such that $h_{0}(x) \neq g_{0}(x)$. Let $V, W$ be disjoint neighborhoods of $h_{0}(x)$ and $g_{0}(x)$, respectively. Then $U=h_{0}^{-1}(V) \cap g_{0}^{-1}(W)$ is a neighborhood of $x$ whose images under $h_{0}$ and $g_{0}$, respectively, are disjoint. Since $\bigcup_{i=1}^{\infty} C_{i}$ is dense, and diam $C_{i} \rightarrow 0$, there is an integer $\mathrm{n}_{0}$ such that $C_{n_{0}} \subset U$, and we have $h_{0}\left(C_{n_{0}}\right) \cap g_{0}\left(C_{n_{0}}\right)=\phi$.

Let $A=\left\{h \in G: h\left(C_{n_{0}}\right)=h_{0}\left(C_{n_{0}}\right)\right\}$ and let $B=G-A$. Then $h_{0} \in A, g_{0} \in B$, and $A \cup B=G$.

We will show that $A$ and $B$ are mutually separate, by showing that there is a $\delta>0$ such that $d(A, B) \geqq \delta$. Let $\delta$ be the diameter of $h_{0}\left(C_{n_{0}}\right)$, and let $h_{1}$ be any element of $A$, and let $g_{1}$ be any element of $B$. Since $h_{1}\left(C_{n_{0}}\right)$ and $g_{1}\left(C_{n_{0}}\right)$ are 2 disjoint circles in $E^{2}$, neither containing the other, there exists a straight line $L$ in $E^{2}$ separating them. (We can obtain $L$ by first constructing the line $L^{\prime}$ joining the centers $a$ and $b$ of $h_{1}\left(C_{n_{0}}\right)$ and $g_{1}\left(C_{n_{0}}\right)$, respectively, and then constructing the line $L$ perpendicular to $L^{\prime}$, with $L$ going through the midpoint of the smallest segment of $[a, b]$ which joins $h_{1}\left(C_{n_{0}}\right)$ and $g_{1}\left(C_{n_{0}}\right)$.) We note that $h_{1}\left(C_{n_{0}}\right)=h_{0}\left(C_{n_{0}}\right)$.

Now let $y$ be the point of $L^{\prime} \cap h_{0}\left(C_{n_{0}}\right)$ which is farther from $L$. Then $y=h_{1}(x)$ for some $x$ in $C_{n_{0}}$. Since $g_{1}(x) \in g_{1}\left(C_{n_{0}}\right)$, and therefore on the other side of $L$, $d\left(h_{1}(x), g_{1}(x)\right)>\delta$. Therefore $d\left(h_{1}, g_{1}\right)>\delta$. We have thus shown that the distance between any element of $A$ and any element of $B$ is greater than $\delta$. It follows that $d(A, B) \geqq \delta$.

TheOREM 1.3 (R. D. ANDERSON). The group of all homeomorphisms of the universal curve $N$ is totally disconnected.

Proof. (See $\$ 3$ for the definitions of universal plane curve and universal curve.)

We leave it to the reader to supply definitions for essential and inessential wrapping used in the proof below.

Let $h_{0}$ be any homeomorphism of $N$ onto itself, $h_{0} \neq e$. Let $x$ be a point of $N \ni h_{0}(x) \neq x$. If we project $x$ and $h_{0}(x)$ onto each of the faces of $N$, they must

(4) This proof was outlined to me by R. D. Anderson. 
be distinct on at least one face, $F . F$ is a universal plane curve. Let $U$ be a neighborhood of $x$ such that (1) $\pi(U) \cap \pi h_{0}(U)=\phi$ and (2) $F-\pi(U)$ and $F-\pi h_{0}(U)$ are each connected, where $\pi$ is the projection of $N$ onto $F$. Let $A$ be a "boundary" square in $\pi(U)$ for which there exists a simple closed curve $B$ in $U$ such that (1) $\pi(B)=A$ and (2) $\pi$ is $1-1$ on $B$.

Let $C$ be the unit circle in $E_{2}$ and let $g: C \rightarrow B$ be any homeomorphism. Since $h_{0} g: C \rightarrow h_{0}(U)$ is a homeomorphism, $h_{0} g(C)$ wraps around $A$ inessentially, because $\pi h_{0} g(C)$ does. Thus $h_{0}(B)$ does, also.

Let $G_{1}=\{h \in G(N) \mid \pi h(B)$ wraps around $A$ essentially $\}$. Let $G_{2}=G(N)-G_{1}$. Then $e \in G_{1}, h_{0} \in G_{2}$, and $G(N)=G_{1} \cup G_{2} . G_{1}$ and $G_{2}$ are each closed. [To see this, we may use Example VI 8, p. 85 of Hurewicz and Wallman's Dimension Theory, to get the limit map of a sequence of essential (or inessential) maps to be essential (or inessential).] Thus $G_{1}$ and $G_{2}$ are mutually separate, and provide a separation of $h_{0}$ from $e$.

Since $G(N)$ is a group, it follows that any pair of homeomorphisms can be separated. Thus $G(N)$ is totally disconnected.

2. Continua with nonzero dimensional groups of homeomorphisms. In this section we establish sufficient conditions for continua to have nonzero dimensional groups of homeomorphisms. Our main result of this section is the following: Let $X$ be a locally setwise homogeneous $\left({ }^{5}\right)$ continuum. Then $G(X)$ is at least 1-dimensional.

Among the continua which are locally setwise homogeneous $\left(^{6}\right)$ are the universal plane curve $\left({ }^{7}\right)$, universal curve $\left({ }^{8}\right)$, and compact manifolds of all positive dimensions. Thus the groups of all homeomorphisms of these continua are at least one dimensional. However, it is easy to show (see Theorem 1.1) that the groups of all homeomorphisms of the manifolds are infinite dimensional. By methods similar to those used in the proof of Theorem 1.1, we see that the groups of homeomorphisms of the universal plane curve and universal curve contain infinite products of nonzero dimensional sets, and are quite possibly infinite dimensional. However, we have not been able to determine precisely the dimensions of these sets.

As a corollary of the proof of the above theorem, it also follows that if $H$ is the group of those homeomorphisms of a compact $n$-manifold, $n>1$, which carry a countable dense subset of the interior of the manifold onto itself, then $H$ is at least 1-dimensional.

We note that, in $\S 5$, we will use a very much simplified version of the proof of Theorem 2.1 to show nonzero dimensionality for the groups of homeomorphisms of the examples of that section, specifically in Theorem 5.1.

(5) See below for definition.

(6) Ibid.

(7) See $\$ 3$ for definition.

(8) Ibid. 
Definition 2.1. A chain of open sets $\mathscr{U}: U_{1}, \cdots, U_{n}$, is a finite, ordered set of open sets such that

(1) $U_{i} \cap U_{j} \neq \phi$ iff $|i-j| \leqq 1$,

(2) $\bar{U}_{i} \cap \bar{U}_{j} \neq \phi$ iff $U_{i} \cap U_{j} \neq \phi$, and

(3) $U_{i} \notin U_{j}$ for any pair $i, j$.

The elements of the chain are called its links.

A subset of $\mathscr{U}$ will be called a subchain if its elements form a chain with the order inherited from $\mathscr{U}$.

A chain whose links are of diameter $<\varepsilon$ is called an $\varepsilon$-chain.

Definition 2.2. Let $\mathscr{U}: U_{1}, \cdots, U_{n}$ be a chain of open sets. Then a set $A=\left\{a_{i}\right\}_{i=0}^{n}$ is called a chain set for $\mathscr{U}$ if

(1) $a_{0} \in U_{1}-U_{2}$,

(2) $a_{i} \in U_{i} \cap U_{i+1}$, for $1 \leqq i \leqq n-1$, and

(3) $a_{n} \in U_{n}-U_{n-1}$.

Definition 2.3. Let $X$ be a continuum, and let $\mathscr{U}: U_{1}, \cdots, U_{n}$ be a chain of connected open sets of $X$. Then a chain $\mathscr{V}: V_{1}, \cdots, V_{m}$ of connected open sets of $X$ is called a straight refinement of $\mathscr{U}$ if

(1) there exists a chain set $A=\left\{a_{i}\right\}_{i=0}^{n}$ for $\mathscr{U}$ such that

(a) $\mathscr{V}$ is a minimal chain from $a_{0}$ to $a_{n}$ with $a_{0} \in V_{1}$ and $a_{n} \in V_{m}$,

(b) $\mathscr{V} *$ contains $A$,

(c) $\overline{\mathscr{V}_{i}^{*}} \subset U_{i}$ where $\mathscr{V}_{i}$ is the shortest subchain of $\mathscr{V}$ from $a_{i-1}$ to $a_{i}$ and,

(d) each $a_{i}$ is an element of the intersection of 2 links of $\mathscr{V}$ and

(2) exactly one link of $\mathscr{V}_{i}$ meets the boundary of $U_{i+1}$.

$A$ is called an associated chain set for $\{\mathscr{U}, \mathscr{V}\}$.

Note 1. (c) of (1) implies that $\mathscr{V}$ is a closed refinement of $\mathscr{U}$.

Note 2. The chain $\mathscr{V}$ in reverse order need not be a straight refinement of the chain $\mathscr{U}$ in reverse order.

Definition 2.4. The chain $\mathscr{V}$ is called a uniform refinement of the chain $\mathscr{U}$ if

(1) $\mathscr{V}$ is a straight refinement of $\mathscr{U}$ and

(2) if $k_{i}$ denotes the number of links in $\mathscr{V}_{i}$ (of definition 3.1.c), then $k_{i}=k$ for $1 \leqq i, j \leqq n$.

The chain $\mathscr{V}_{i}$ is called the $i$ th uniform subchain of $\mathscr{V}$. (We note that $\mathscr{V}_{i}$ depends on the associated chain set for $\{\mathscr{U}, \mathscr{V}\}$ chosen, only if $\mathscr{V}$ is not a uniform refinement.)

Definition 2.5. Let $X$ be a continuum, $\mathscr{U}: U_{1}, \cdots, U_{n}$ be a chain of connected open sets of $X$. A homeomorphism $h$ of $X$ onto itself, $h$ supported on $\mathscr{U}^{*}$, is called a pseudo translation of $k$ units on $\mathscr{U}$ if there exists a chain set $A=\left\{a_{i}\right\}_{i=0}^{n}$ for $\mathscr{U}$ such that

$$
h\left(a_{i}\right)=a_{i+k}, \quad \text { for } 0 \leqq i \leqq n-k,
$$

$$
h\left(a_{i}\right) \in U_{n}, \quad \text { for } i>n-k,
$$




$$
\begin{array}{ll}
h\left(\bar{U}_{i}\right) \subset\left[\left(\bigcup_{j=i-1}^{i+k} \bar{U}_{j}\right)-\bar{U}_{i-2}\right], & \text { for } 1 \leqq i \leqq n-k, \\
h\left(\bar{U}_{i}\right) \subset\left[\left(\bigcup_{j \geqq i-1} \bar{U}_{j}\right)-\bar{U}_{i-2}\right], & \text { for } i>n-k .
\end{array}
$$

$A$ is called a chain set for $\mathscr{U}$ associated with $h$.

For any $\varepsilon>0$, we call $h$ an $\varepsilon$-pseudo translation if (2) can be strengthened to:

$$
\begin{array}{ll}
h\left(\bar{U}_{i}\right) \subset\left[\left(\bigcup_{j=i}^{i+k} \bar{U}_{j}\right) \cup N_{\varepsilon}\left(U_{i}\right)\right], & \text { for } 1 \leqq i \leqq n-k ; \\
h\left(\bar{U}_{i}\right) \subset\left[\left(\bigcup_{j \geqq i} \bar{U}_{j}\right) \cup N_{\varepsilon}\left(U_{i}\right)\right], & \text { for } i>n-k,
\end{array}
$$

where $N_{\varepsilon}\left(U_{i}\right)$ denotes the $\varepsilon$-neighborhood of $U_{i}$.

Definition 2.6. Let $X$ be a continuum, and let $\mathscr{U}: U_{1}, \cdots, U_{n}$ be a chain of connected open sets of $X$. Then a homeomorphism $h$ of $X$ onto itself, $h$ supported on $\mathscr{U}^{*}$, is called a sliding homeomorphism of $k$ units on $\mathscr{U}$ if there exists a chain $\mathscr{D}: D_{1}, \cdots, D_{n-k}$ of connected open sets such that

(1) $D_{i} \subset U_{i+k}$,

(2) $h^{-1}\left(D_{i}\right) \subset U_{i}$ and

$$
\begin{array}{ll}
h\left(\bar{U}_{i}\right) \subset\left[\left(\bigcup_{j=i-1}^{i+k} \bar{U}_{j}\right)-\bar{U}_{i-2}\right], & \text { for } 1 \leqq i \leqq n-k ; \\
h\left(\bar{U}_{i}\right) \subset\left[\left(\bigcup_{j \geqq i-1} \bar{U}_{j}\right)-\bar{U}_{i-2}\right], & \text { for } i>n-k .
\end{array}
$$

$\mathscr{D}$ is called an associated chain for $h$.

For any $\varepsilon>0$, we call $h$ an $\varepsilon$-sliding homeomorphism of $k$ units on $\mathscr{U}$ if (3) can be strengthened to:

$$
\begin{array}{ll}
h\left(\bar{U}_{i}\right) \subset\left[\left(\bigcup_{j=i}^{i+k} \bar{U}_{j}\right) \cup N_{\varepsilon}\left(U_{i}\right)\right], & \text { for } 1 \leqq i \leqq n-k ; \\
h\left(\bar{U}_{i}\right) \subset\left[\left(\bigcup_{j \geqq i} \bar{U}_{j}\right) \cup N_{\varepsilon}\left(U_{i}\right)\right], & \text { for } i>n-k,
\end{array}
$$

where $N_{\varepsilon}\left(U_{i}\right)$ denotes the $\varepsilon$-neighborhood of $U_{i}$.

We call $h$ a weak sliding homeomorphism if it satisfies condition (3) above, but not necessarily (1) and (2).

Definition 2.7. Let $X$ be a continuum, and let $H$ be a subgroup of $G(X)$. Then $X$ is called locally setwise homogeneous under $H$ if there exist both a basis, $\mathscr{E}$, of connected open subsets of $X$, and also a dense subset, $B$, of $X$ such that for any element $E$ of $\mathscr{E}$, and any 2 points $a$ and $b$ of $B \cap E$, there exists a homeomorphism $h \in H, h$ supported on $E$, such that $h(a)=b$. 
If $X$ is locally setwise homogeneous under $G(X)$, then $X$ is called locally setwise homogeneous.

We will call $\{X, \mathscr{E}, B, H\}$ a locally setwise homogeneous structure.

REMARK. A locally setwise homogeneous continuum must be locally connected.

Notation: If $\mathscr{C}: C_{1}, \cdots, C_{n}$ is a chain of subsets of $X$, then $h(\mathscr{C})$ will represent the chain of subsets of $X$ whose links are the sets $h\left(C_{i}\right), 1 \leqq i \leqq n$.

LEMMA 2.1. Let $X$ be a locally connected continuum, and let $\mathscr{U}: U_{1}, \cdots, U$ be a chain of connected open sets of $X$. Then for each $\varepsilon>0$, there exists an $\varepsilon-$ chain $\mathscr{V}$ which is a straight refinement of $\mathscr{U}$. The elements of $\mathscr{V}$ can be chosen from any given basis of connected open sets of $X$.

Proof. Standard.

LEMMA 2.2. Let $X$ be a locally connected continuum, and let $\mathscr{U}: U_{1}, \cdots, U_{n}$ be a chain of connected open sets of $X$. Then for each $\varepsilon>0$, there exists a uniform $\varepsilon$-refinement $\mathscr{W}$ of $\mathscr{U}$.

Proof. Let $\delta=\min _{i}\left\{d\left(U_{i}, U_{i+2}\right)\right\}$ and let $\gamma<\min \{\delta / 3, \varepsilon\}$. By Lemma 2.1, there exists a $\gamma$-chain $\mathscr{V}$ which is a straight refinement of $\mathscr{U}$. Let $A=\left\{a_{i}\right\}_{i=0}^{n}$ be an associated chain set for $\{\mathscr{U}, \mathscr{V}\}$.

Let $k_{i}$ be the number of links in $\mathscr{V}_{i}$, the $i$ th subchain of $\mathscr{V}$, and let $k=\max _{i}\left\{k_{i}\right\}$. We will modify $\mathscr{V}$ to a chain $\mathscr{W}$ which has the property that, for all $i$, the number of links in $\mathscr{W}_{i}$ is $k$.

For each $i$ for which $\mathscr{V}_{i}$ contains less than $k$ links, we choose a link $V_{t_{i}}$ such that $V_{t_{i}} \subset U_{i}$, but $V_{t_{i}} \cap U_{j}=\phi$ for $j \neq i$. Let $\alpha=\min _{i}\left\{d\left(V_{i-1}, V_{i+1}\right)\right\}$. Let $k^{\prime}=\max _{i}\left\{k-k_{i}\right\}$, and let $b_{i} \in V_{t_{i}-1} \cap V_{t_{i}}$ and $b_{i}^{\prime} \in V_{t_{i}} \cap V_{t_{i}+1}$. Let $\mathscr{C}_{i}$ be a chain of connected open sets from $b_{i}$ to $b_{i}^{\prime}$ of mesh $<\alpha / k^{\prime}$, whose closures are in $V_{t_{i}}$. Let $\mathscr{C}_{i}^{\prime}$ be the subchain of $\mathscr{C}_{i}$ from the last link of $\mathscr{C}_{i}$ which meets $V_{t_{i}-1}$ to the first link of $\mathscr{C}_{i}$ which meets $V_{t_{i}+1}$. Then $\mathscr{C}_{i}^{\prime}$ contains at least $k^{\prime}+1$ elements. Next, we modify $\mathscr{C}_{i}^{\prime}$ to a chain $\mathscr{C}_{i}^{\prime \prime}$ whose links are obtained from $\mathscr{C}_{i}^{\prime}$ by taking, as one link, the union of all but the first $k-k_{i}$ links, and by taking the first $k-k_{i}$ links of $\mathscr{C}_{i}^{\prime}$ as links of $\mathscr{C}_{i}^{\prime \prime}$ also. Then let $\mathscr{W}_{i}$ be the chain consisting of all links of $\mathscr{V}_{i}$ except $V_{t_{i}}$ which is to be replaced by $\mathscr{C}_{i}{ }^{\prime \prime}$. Then $\mathscr{W}_{i}$ has exactly $k$ links. Let $\mathscr{W}$ be the chain consisting of the collection of links of the $\mathscr{W}_{i}$ 's. Then $\mathscr{W}$ is the desired chain.

LEMMA 2.3. Let $\{X, \mathscr{E}, B, H\}$ be a locally setwise homogeneous structure, let $\mathscr{U}: U_{1}, \cdots, U_{n}$ be a chain of connected open sets of $X$, and let $\varepsilon>0$. Then for each integer $k, 1 \leqq k \leqq n$, there exists an $\varepsilon$-pseudo translation in $H$ of $k$ units on $\mathscr{U}$.

Proof. Let $\delta=\min _{i}\left\{d\left(U_{i}, U_{i+2}\right), \varepsilon\right\}$. Let $\mathscr{V}$ be a straight refinement of $\mathscr{U}$ of mesh $<\delta / n$ and whose links are elements of $\mathscr{E}$. By Lemma 2.1, $\mathscr{V}$ exists. Let 
$A=\left\{a_{i}\right\}_{i=0}^{n}$ be a chain set for $\{\mathscr{U}, \mathscr{V}\}$, with $A \subset B$. (Since $B$ is dense and the $a_{i}$ 's are chosen in open sets, we can get $A \subset B$.)

Let $\mathscr{V}_{i}: V_{i, 1}, \cdots, V_{i, k_{i}}$ be the shortest subchain of $\mathscr{V}$ from $a_{i-1}$ to $a_{i}$ in $U_{i}$. For each $i, 1 \leqq i \leqq n$, let $\phi_{i, 1}$ be a homeomorphism supported on $V_{i, 1}, \phi_{i, 1} \in H$, and carrying $a_{i-1}$ to some point of $V_{i, 2}$. We define $\phi_{i, j}$ for $1<j<k_{i}$ inductively by: $\phi_{i, j}$ is a homeomorphism $\in H$ supported on $V_{i, j}$ and carrying $\phi_{i, j-1} \cdots \cdots \phi_{i, 2} \phi_{i, 1}\left(a_{i-1}\right)$ to some point of $V_{i, j+1}$. For $j=k_{i}, \phi_{i, j} \in H$ is supported on $V_{i, j}$ and

$$
\phi_{i, j}\left(\phi_{i, j-1} \cdots \cdots \phi_{i, 1}\left(a_{i-1}\right)\right)=a_{i} .
$$

Now let $h_{i}=\phi_{i, s_{i}-1} \cdots \phi_{i, 1}$ where $V_{i, s_{i}}$ is the first link of $\mathscr{V}_{i}$ meeting $U_{i+1}$, for $1 \leqq i<n$, and let $h_{n}=\phi_{n, k_{n}} \cdots \cdots \phi_{n, 2} \phi_{n, 1}$. Let $g_{i}=\phi_{i, k_{i}} \cdots \cdot \phi_{i, s_{i}}$, for $1 \leqq i<n$. Let $h=\prod_{i=1}^{n-1} g_{i} \prod_{i=1}^{n} h_{i}$. Then $h$ is a $\delta / n$-pseudo translation of 1 unit in $H$. Further, for this $h, h^{k}$ is a $[k \delta / n(<\varepsilon)]$ pseudo translation of $k$ units on $\mathscr{U}$, since by this choice of $h_{i}$, no point can be moved back more than 1 link of $\mathscr{V}$.

Lemma 2.4. Let $X$ be a locally setwise homogeneous continuum, $\mathscr{U}: U_{1}, \cdots, U_{n}$ be a chain of connected open sets of $X$, and $h$ be a pseudo translation of $k$ units on $\mathscr{U}$. Then there exists a chain $\mathscr{D}: D_{1}, \cdots, D_{n-k}$, of connected open sets of $X$ such that (1) $D_{i} \subset U_{i}$ and (2) $h\left(D_{i}\right) \subset U_{i+k}$. Thus it follows that the chain $h(\mathscr{D})$ satisfies the following conditions: (1) $h\left(D_{i}\right) \subset U_{i+k}$ and (2) $h^{-1}\left(h\left(D_{i}\right)\right) \subset U_{i}$; that is, $h$ is a sliding homeomorphism of $k$ units on $\mathscr{U}$.

Proof. Let $A=\left\{a_{i}\right\}_{i=0}^{n}$ be a chain set for $\mathscr{U}$ associated with $h$. For $i$ such that $1 \leqq i \leqq n-k$, let

$$
C_{i}=\bar{U}_{i} \cap h^{-1}\left[\left(\bigcup_{j=-1}^{k-1} \bar{U}_{i+j}\right)-U_{i+k}\right] .
$$

Then $C_{i}$ is a closed subset of $X$ and $C_{i}^{\prime}=C_{i} \cap U_{i}$ is a closed subset of $U_{i}$. Let $D_{i}^{\prime}=U_{i}-C_{i}^{\prime}$. Then $D_{i}^{\prime}$ is an open subset of $U_{i}$, and therefore of $X$, and $h\left(D_{i}^{\prime}\right) \subset U_{i+k}$. We note that $D_{i}^{\prime}$ contains both $a_{i-1}$ and $a_{i}$. Further, $C_{i}^{\prime}$ cannot separate $a_{i-1}$ from $a_{i}$, for if it did, then $h\left(C_{i}^{\prime}\right)$ would separate $\left(h\left(a_{i-1}\right)\right.$ from $h\left(a_{i}\right)$ that is, $a_{i+k-1}$ from $a_{i+k}$. But $h\left(C_{i}^{\prime}\right) \cap U_{i+k}=\phi$. This is a contradiction. Let $D_{i}$ be the component of $D_{i}^{\prime}$ which contains both $a_{i-1}$ and $a_{i}$. Then $D_{i} \subset U_{i}$ and $h\left(D_{i}\right) \subset U_{i+k}$.

LEMMA 2.5. Let $\{X, \mathscr{E}, B, H\}$ be a locally setwise homogeneous structure, and let $\mathscr{U}: U_{1}, \cdots, U_{n}$ be a chain of connected open sets of $X$ of mesh $<\varepsilon$. Let $h$ be a sliding homeomorphism of $k$ units on $\mathscr{U}$, and let $\mathscr{D}: D_{1}, \cdots, D_{n-k}$ be an associated chain for $h$; that is, (1) $D_{i} \subset U_{i+k}$ and (2) $h^{-1}\left(D_{i}\right) \subset U_{i}$. Let $\phi$ be a $\delta$-sliding homeomorphism of 1 unit on $\mathscr{D}$, where $2 \delta$ is less than $\min _{i}\left\{d\left(\bar{U}_{i-2}\right.\right.$, $\left.\left.h\left(\bar{U}_{i}\right) \cup \bar{U}_{i}\right)\right\}$, and $\phi$ is constructed as $h$ is constructed in Lemma 2.3. Then

(1) $\phi h$ is a sliding homeomorphism of $k+1$ units on $\mathscr{U}$, and

(2) $d(h, \phi h)<3 \varepsilon$. 
Proof. (1) Let $\mathscr{E}: E_{1}, \cdots, E_{n-k-1}$ be an associated chain for $\phi$. Then $\mathscr{E}$ is also an associated chain for $\phi h$.

(2) Clear.

LEMMA 2.6. Let $X$ be a continuum, and let $h$ be a sliding homeomorphism of $k$ units on a chain $\mathscr{U}: U_{1}, \cdots, U_{n}$ of connectecd open sets of $X$. Let $\mathscr{D}: D_{1}, \cdots, D_{n-k}$ be an associated chain for $h$. Let $2 \varepsilon=\min _{i \geqq 3}\left\{d\left(\bar{U}_{i-2}, h\left(\bar{U}_{i}\right) \cup \bar{U}_{i}\right)\right\}$. Let $\mathscr{V}: V_{1}, \cdots, V_{s} \cdot(n-k)$ be a uniform refinement of $\mathscr{D}$ of mesh $<\varepsilon$, and let $\phi$ be a sliding homeomorphism of $s$ units on $\mathscr{V}$. Then $\phi h$ is a sliding homeomorphism of $k+1$ units on $\mathscr{U}$.

Proof. Let $\mathscr{E}: E_{1}, \cdots, E_{s} \cdot(n-k-1)$ be an associated chain for $\phi$. We form a new chain $\mathscr{F}: F_{1}, \cdots, F_{n-k-1}$ by an amalgamation process from $\mathscr{E}$, by letting $\left.F_{i}=\bigcup_{j=[s}^{s \cdot i} \cdot(i-1)\right]+1 E_{j}$. Then $F_{i} \subset U_{i+k+1}$ and $\mathscr{F}$ is an associated chain for $\phi h$. This is easy to see since $\phi^{-1}\left(F_{i}\right) \subset D_{i} \subset U_{i+k}$ and therefore $h^{-1}\left(\phi^{-1}\left(F_{i}\right)\right) \subset h^{-1}\left(D_{i}\right) \subset U_{i}$; that is, $(\phi h)^{-1}\left(F_{i}\right) \subset U_{i}$. It is also clear that

$$
\phi h\left(\bar{U}_{i}\right) \subset\left[\left(\bigcup_{j=i-1}^{i+k+1} \bar{U}_{j}\right)-\bar{U}_{i-2}\right] .
$$

Thus $\phi h$ is a sliding homeomorphism of $k+1$ units on $\mathscr{U}$.

Lemma 2.7. Let $X$ be a continuum, and let $\mathscr{U}: U_{1}, \cdots, U_{n}$ be a chain of connected open subsets of $X$ of mesh $<\varepsilon$. Then if $h$ is any homeomorphism of $X$ onto itself, and $\phi$ is a homeomorphism supported on $\mathscr{U}^{*}$ such that

$$
\phi\left(U_{i}\right) \subset\left(U_{i-1} \cup U_{i} \cup U_{i+1}\right),
$$

then $d(h, \phi h)<3 \varepsilon$.

Proof. Clear.

LEMMA 2.8. Let $X$ be a continuum, let $\left\{\varepsilon_{n}\right\}_{n=1}^{\infty}$ be a decreasing sequence of positive numbers such that $\sum \varepsilon_{n}$ exists, and let $\left\{s_{n}\right\}_{n=1}^{\infty},\left\{t_{n}\right\}_{n=2}^{\infty}$, and $\left\{k_{n}\right\}_{n=1}^{\infty}$ be sequences of integers. Also let $\left\{\mathscr{C}_{n}\right\}_{n=1}^{\infty},\left\{\phi_{n}\right\}_{n=1}^{\infty}$, and $\left\{h_{n}\right\}_{n=1}^{\infty}$ be sequences such that

(1) $\mathscr{C}_{n}: C_{n, 1}, \cdots, C_{n, s_{n}}$ is an $\varepsilon_{n}^{\prime}$-chain of $s_{n}$ connected open sets, where $\varepsilon_{n}^{\prime}<\min \left\{\varepsilon_{n}, \min _{i}\left\{d\left(C_{n-1, i-2}, C_{n-1, i}\right)\right\}\right\}$, and $\varepsilon_{1}^{\prime}=\varepsilon_{1}$,

(2) $\mathscr{C}_{n+1}$ is a uniform refinement of a subchain of $\mathscr{C}_{n}$, with $t_{n+1}$ elements of $\mathscr{C}_{n+1}$ in each element of $\mathscr{C}_{n}$,

(3) for $n \geqq 2, \phi_{n-1}$ is a sliding homeomorphism of $k_{n}$ units on $\mathscr{C}_{n}$, where $k_{n}<t_{n}$ and thus is a weak sliding homeomorphism of 1 unit on $\mathscr{C}_{n-1}$,

(4) $h_{1}$ is a sliding homeomorphism of $k_{1}$ units on $\mathscr{C}_{1}$, and for $n \geqq 2$, $h_{n}=\phi_{n-1} h_{n-1}^{*}$,

(5) $h_{n-1}^{-1}\left(\mathscr{C}_{n}^{*}\right)$ has mesh $<\varepsilon_{n}$, for $n \geqq 2$, and

(6) $\phi_{n-1}^{-1}\left(\mathscr{C}_{n+1}^{*},{ }_{i}\right) \subset C_{n, i}$, for $1 \leqq i \leqq s_{n}-k_{n}$, where $\mathscr{C}_{n+1},{ }_{i}$ is the ith uniform subchain of $\mathscr{C}_{n+1}$. 
Then (1) $\left\{h_{n}\right\}$ is Cauchy and

(2) the limit map $h$ is a homeomorphism of $X$ onto itself.

Proof. To show that $\left\{h_{n}\right\}$ is Cauchy, we note that $h_{n}=\phi_{n-1} h_{n-1}$ where $\phi_{n-1}$ is supported on $\mathscr{C}_{n}$ and is a weak sliding homeomorphism of 1 unit on $\mathscr{C}_{n-1}$. By Lemma 2.7, $d\left(h_{n-1}, \phi_{n-1} h_{n-1}\right)<3 \varepsilon_{n-1}$, and as $h_{n}=\phi_{n-1} h_{n-1}$, we have $d\left(h_{n-1}, h_{n}\right)<3 \varepsilon_{n-1}$. But $\sum \varepsilon_{n}<\infty$; therefore $\left\{h_{n}\right\}$ is Cauchy. Since this is a Cauchy sequence of homeomorphisms of a compact space, $\left\{h_{n}\right\}$ has a limit map $h[6$, p. 56]. We note that $h$ is the infinite product $\cdots \phi_{3} \phi_{2} \phi_{1} h_{1}$.

We show that $h$ is onto. Let $x_{0} \in X$, and let $a_{n}=h_{n}^{-1}\left(x_{0}\right)$. We assume that $\left\{a_{n}\right\}$ converges to some point $a$ of $X$; (if not, we choose a convergent subsequence). Then $h_{n}\left(a_{n}\right)=x_{0}$. We show that $h(a)=x_{0}$ also. Let $\gamma_{i} \rightarrow 0$. Since $h_{n} \rightarrow h$, for each $\gamma_{i}$ there exists an integer $n_{i}$ such that $d\left(h_{n_{i}}, h\right)<\gamma_{i}$. Therefore $d\left(h_{n_{i}}\left(a_{n_{i}}\right), h\left(a_{n_{i}}\right)\right)<\gamma_{i}$; that is, $d\left(x_{0}, h\left(a_{n_{i}}\right)\right)<\gamma_{i}$. But this means that $h\left(a_{n_{i}}\right) \rightarrow x_{0}$. Now $\left\{a_{n_{i}}\right\}$ is a subsequence of $\left\{a_{n}\right\}$. Therefore $a_{n_{i}} \rightarrow a$. By the continuity of $h, h\left(a_{n_{i}}\right) \rightarrow h(a)$. It follows that $x_{0}=h(a)$. Therefore $h$ is onto.

To show that $h$ is a homeomorphism, it is sufficient, since $X$ is compact, to show that $h$ is $1-1$.

We first observe that $\bigcap_{n} \mathscr{C}_{n}{ }^{*}$ is an arc $[7$, p. 84], or a point. We then note that $h$ carries $\bigcap_{n} h_{n-1}^{-1}\left(\mathscr{C}_{n}^{*}\right)\left(=\bigcap_{n} h_{n}^{-1}\left(\mathscr{C}_{n}^{*}\right)\right)$ onto $\bigcap_{n} \mathscr{C}^{*}$. This is true since for each $n$ and each $m>n, h_{m}$, as well as $h_{n}$, carries $h_{n}^{-1}\left(\mathscr{C}_{n}^{*}\right)$ onto $\mathscr{C}_{n}^{*}$. Thus $h$ also carries $h_{n}^{-1}\left(\mathscr{C}_{n}^{*}\right)$ onto $\mathscr{C}_{n}^{*}$. It follows that $h$ carries $\bigcap_{n} h_{n}^{-1}\left(\mathscr{C}_{n}^{*}\right)$ onto $\bigcap_{n} \mathscr{C}_{n}^{*}$, and as $\bigcap_{n} h_{n}^{-1}\left(\mathscr{C}_{n}^{*}\right)=\bigcap_{n} h_{n-1}^{-1}\left(\mathscr{C}_{n}^{*}\right), h\left[\bigcap_{n} h_{n-1}^{-1}\left(\mathscr{C}_{n}^{*}\right)\right]=\bigcap_{n} \mathscr{C}_{n}^{*}$.

We next observe that if $x_{0} \notin \bigcap_{n} h_{n-1}^{-1}\left(\mathscr{C}_{n}^{*}\right)$ then $h\left(x_{0}\right)$ is determined at a finite stage. For there exists an integer $N$ such that $x_{0} \notin h_{N-1}^{-1}\left(\mathscr{C}_{N}^{*}\right)$. Therefore $h_{N-1}\left(x_{0}\right) \notin \mathscr{C}_{N}^{*}$. But $h=\phi h_{N-1}$ where $\phi$ is supported on $\mathscr{C}_{N}^{*}$. Thus $h\left(x_{0}\right)$ $=h_{N-1}\left(x_{0}\right)=h_{n}\left(x_{0}\right)$ for all $n>N-1$, and $h\left(x_{0}\right)$ is determined at a finite stage.

Now, to show that $h$ is $1-1$, we consider 3 cases. If $x \neq y, x, y \notin \bigcap_{n} h_{n-1}^{-1}\left(\mathscr{C}_{n}^{*}\right)$, then by the above comments, $h(x)$ and $h(y)$ are determined at finite stages; that is, there exist integers $M, N$ such that $h(x)=h_{M}(x)=h_{m}(x)$ for $m>M$, and $h(y)=h_{N}(y)=h_{n}(y)$ for $n>N$. Let $R=\max \{M, N\}$. Then $h_{R}(x)=h(x)$ and $h_{R}(y)=h(y)$. Since $h_{R}$ is a homeomorphism, $h_{R}(x) \neq h_{R}(y)$; that is, $h(x) \neq h(y)$.

If $x \neq y, x \notin \bigcap{ }_{n} h_{n-1}^{-1}\left(\mathscr{C}_{n}^{*}\right)$ and $y \in \bigcap_{n} h_{n-1}^{-1}\left(\mathscr{C}_{n}^{*}\right)$, then by the above comments $h(x) \notin \bigcap_{n} \mathscr{C}_{n}^{*}$, but $h(y) \in \bigcap_{n} \mathscr{C}_{n}^{*}$. So again, $h(x) \neq h(y)$.

Finally, if $x \neq y, x, y \in \bigcap_{n} h_{n-1}^{-1}\left(\mathscr{C}_{n}^{*}\right)$, let $\delta=d(x, y)$. Since for each $n$, the mesh of $h_{n-1}^{-1}\left(\mathscr{C}_{n}\right)<\varepsilon_{n}$, and $\varepsilon_{n} \rightarrow 0$, there is an $N$ such that $x$ and $y$ are separated by at least 8 elements of $h_{N-1}^{-1}\left(\mathscr{C}_{N}\right)$. Therefore $h_{N-1}(x)$ and $h_{N-1}(y)$ are separated by at least 8 elements of $\mathscr{C}_{N}$. Now $h_{N}$ is $\phi_{N-1} h_{N-1}$ where $\phi_{N-1}$ is a sliding homeomorphism of $k_{N}$ units on $\mathscr{C}_{N}$ and, by (6) $\phi_{N-1}^{-1}\left(\mathscr{C}_{N+1, i}^{*}\right) \subset C_{N, i}$. Further, $\phi_{N-1}\left(h_{N-1}(x)\right)$ and $\phi_{N-1}\left(h_{N-1}(y)\right)$ are both in $\mathscr{C}_{N+1}^{*}$; that is, $h_{N}(x)$ and $h_{N}(y)$ are in $\mathscr{C}_{N+1}^{*}$. Thus $h_{N-1}(x)$ and $h_{N-1}(y)$ must each be carried at least $k_{N}-1$ units of $\mathscr{C}_{N}$ 
to the right and at most $k_{N}+1$ units of $\mathscr{C}_{N}$ to the right. Thus $h_{N}(x)$ and $h_{N}(y)$ are separated by at least 6 elements of $\mathscr{C}_{N}$.

Now $h=\phi h_{N}$ where $\phi$ is supported on $\mathscr{C}_{N+1}^{*}$ and is a weak sliding homeomorphism of at most 2 units on $\mathscr{C}_{N}$. This is true since $\phi=\cdots \phi_{N+2} \phi_{N+1} \phi_{N}$, where $\phi_{i}$ is a weak sliding homeomorphism of 1 unit on $\mathscr{C}_{i}$. Thus $\phi_{N}$ can carry $h_{N}(x)$ at most one element of $\mathscr{C}_{N}$ to the right. Now $\phi_{N+1}$ can carry $\phi_{N}\left(h_{N}(x)\right)$ at most two units of $\mathscr{C}_{N+1}$ to the right but no more than one more unit of $\mathscr{C}_{N}$ to the right. Similarly $\phi_{N+2}$ can carry $\phi_{N+1}\left(\phi_{N}\left(h_{N}(x)\right)\right)$ at most two units of $\mathscr{C}_{N+1}$ to the right and add at most "one-half unit" of $\mathscr{C}_{N}$ to the right. In general, $\phi_{N+j}$ will move $\phi_{N+j-1} \cdots \phi_{N} h_{N}(x)$ at most $1 / 2^{j-1}$ of a unit of $\mathscr{C}_{N}$ to the right. Thus, in the limit, $\phi$ can move $h_{N}(x)$ at most three units to the right. Similarly $\phi$ can move $h_{N}(y)$ at most two units to the left. Thus $\phi h_{N}(x)$ and $\phi h_{N}(y)$ are separated by at least one element of $\mathscr{C}_{N}$, and therefore $h(x) \neq h(y)$.

Therefore $h$ is $1-1$, and thus a homeomorphism of $X$ onto itself.

THEOREM 2.1. Let $X$ be a continuum which is locally setwise homogeneous under the closed subgroup $H$ of $G$. Then $H$ is at least 1-dimensional.

Proof. We will show that for sufficiently small $\varepsilon>0$, every neighborhood $U$ of the identity has a point $h \in H$ on its boundary. We do this by obtaining a sequence of pairs of homeomorphisms of $H,\left\{h_{n}, g_{n}\right\}$, such that

(1) $h_{n} \in U, g_{n} \in C(U)$,

(2) $d\left(h_{n}, g_{n}\right)<3 \varepsilon_{n}, \varepsilon_{n} \rightarrow 0$, and

(3) $\left\{h_{n}\right\}$ satisfies the conditions of Lemma 2.8 .

Then

(1) $\left\{h_{n}\right\}$ is Cauchy,

(2) the limit map $h$ is a homeomorphism, and

(3) $h \in \mathrm{Bd} U$.

Since $H$ is closed, $h \in H$, and therefore $H$ is at least 1-dimensional.

Let $g$ be any homeomorphism of $X$ onto itself, $g \neq e$. Let $x_{0}$ be a point of $X$ such that $g\left(x_{0}\right) \neq x_{0}$, and let $\varepsilon=d\left(x_{0}, g\left(x_{0}\right)\right)$. Let $U$ be a neighborhood of the identity such that diam $U<\varepsilon / 3$. We will show that $H \cap \operatorname{Bd} U \neq \phi$.

Let $\left\{\varepsilon_{n}\right\}_{n=1}^{\infty}$ be a decreasing sequence of positive numbers such that (1) $\varepsilon_{1}<\varepsilon / 3$ and (2) $\Sigma_{n} \varepsilon_{n}<\infty$. Let $\mathscr{C}_{1}: C_{1,1}, C_{1,2}, \cdots, C_{1, s_{1}}$ be an $\varepsilon_{1}$-chain of connected open sets of $X$ from $x_{0}$ to $g\left(x_{0}\right)$, and let $s_{1}$ be the number of links of $\mathscr{C}_{1}$. Let $h_{1}$ be a sliding homeomorphism in $H$ of the largest possible number of units $\left(k_{1}\right)$ on $\mathscr{C}_{1}$ such that $h_{1} \in U$. (We observe that $k_{1}<s_{1}-2$, for any sliding homeomorphism of $s_{1}-2$ units moves some point of $C_{1,1}$ to some point of $C_{1, s_{1}}$, and therefore is of distance more than $\varepsilon / 3$ from the identity. Thus such a homeomorphism is in $C(U)$.) Since $h_{1}$ is a sliding homeomorphism of $k_{1}$ units on $\mathscr{C}_{1}$, there exists a chain $\mathscr{D}_{1}: D_{1,1}, \cdots, D_{1, s_{1}-k_{1}}$, whose links are connected open sets such that 
(1) $D_{1, i} \subset C_{1, i+k_{1}}$, and

(2) $h_{1}^{-1}\left(D_{1, i}\right) \subset C_{1, i}$.

Let $\psi_{1}$ be a $\gamma_{1}$-sliding homeomorphism in $H$ of 1 unit on $\mathscr{D}_{1}$, where $2 \gamma_{1}<\min _{i}\left\{d\left(\bar{C}_{1, i-2}, h\left(\bar{C}_{1, i}\right) \cup \bar{C}_{i}\right)\right\}$, constructed as in Lemma 2.5. Then $\psi_{1} h_{1}$ is a sliding homeomorphism of $k_{1}+1$ units on $\mathscr{C}_{1}$, and thus is in $C(U)$. Let $g_{1}=\psi_{1} h_{1}$. By Lemma 2.7, $d\left(h_{1}, g_{1}\right)<3 \varepsilon_{1}$. Then $h_{1} \in U, g_{1} \in C(U)$, and $d\left(h_{1}, g_{1}\right)<3 \varepsilon_{1}$.

Now $\varepsilon_{2}>0$. By the uniform continuity of $h_{1}^{-1}$, there exists a $\delta_{1}>0$ such that $h_{1}^{-1}$ takes any set of diameter less than $\delta_{1}$ to a set of diameter less than $\varepsilon_{2}$. Let $\mathscr{C}_{2}: C_{2,1}, \cdots, C_{2, s_{2}}$ (where $\left.s_{2}=t_{2} \cdot\left(s_{1}-k_{1}\right)\right)$ be a chain of connected open sets of mesh less than $\min \left\{\gamma_{1}, \delta_{1}, \varepsilon_{2}\right\}$, which is a uniform refinement of $\mathscr{D}_{1}$.

Let $\phi_{1}$ be a sliding homeomorphism in $H$ of the largest possible number of units $k_{2}<t_{2}$ on $\mathscr{C}_{2}$ such that $\phi_{1} h_{1}$ is an element of $U$. We note that, by Lemma 2.6, if $\phi$ is a sliding homeomorphism of $t_{2}$ units on $\mathscr{C}_{2}$, then $\phi h_{1}$ is a sliding homeomorphism of $k_{1}+1$ units on $\mathscr{C}_{1}$, and therefore must be in $C(U)$. Let $h_{2}=\phi_{1} h_{1}$.

Since $\phi_{1}$ is a sliding homeomorphism of $k_{2}$ units on $\mathscr{C}_{2}$, there exists a chain $\mathscr{D}_{2}: D_{2,1}, \cdots, D_{2, s_{2}-k_{2}}$, whose links are connected open sets, such that

(1) $D_{2, i} \subset C_{2, i+k_{2}}$, and

(2) $\phi_{1}^{-1}\left(D_{2, i}\right) \subset C_{2, i}$.

Let $\psi_{2}$ be a $\gamma_{2}$-sliding homeomorphism in $H$ of 1 unit on $\mathscr{D}_{2}$, where $2 \gamma_{2}<\min _{i}\left\{d\left(\bar{C}_{2, i-2}, h_{2}\left(\bar{C}_{2, i}\right) \cup \bar{C}_{2, i}\right)\right\}$ constructed as in Lemma 2.5. Then $\psi_{2} \phi_{1}$ is a sliding homeomorphism of $k_{2}+1$ units on $\mathscr{C}_{2}$, and thus $\psi_{2} \phi_{1} h_{1}=\psi_{2} h_{2}$ is in $C(U)$. Let $g_{2}=\psi_{2} h_{2}$. By Lemma 2.5, $d\left(h_{2}, g_{2}\right)<3 \varepsilon_{2}$. Then $h_{2} \in U, g_{2} \in C(U)$, and $d\left(h_{2}, g_{2}\right)<3 \varepsilon_{2}$.

We continue the above process inductively, thus obtaining sequences of integers, $\left\{k_{n}\right\}$ and $\left\{s_{n}\right\}$, and sequences $\left\{\mathscr{C}_{n}\right\},\left\{\phi_{n}\right\}$, and $\left\{h_{n}\right\}$ which satisfy the hypotheses of Lemma 2.8. Then $\left\{h_{n}\right\}$ is Cauchy, and converges to a limit homeomorphism $h$. Since $d\left(h_{n}, g_{n}\right) \rightarrow 0, g_{n} \rightarrow h$ also. Therefore $h \in \mathrm{Bd} U$. Since $H$ is closed, and each $h_{i} \in H, h \in H$. It follows that $H$ is at least 1-dimensional.

COROLLARY 2.1.1. If $X$ is a locally setwise homogeneous continuum, then $G(X)$ is at least 1-dimensional.

Proof. Clear.

Corollary 2.1.2. Let $X$ be a continuum which contains an open set $U$ whose closure $\bar{U}$ is locally setwise homogeneous. Then $G(X)$ is at least 1-dimensional.

Proof. Let $G^{\prime}$ be the group of all homeomorphisms of $\bar{U}$ onto itself, and let $H^{\prime}$ be the subgroup of $G^{\prime}$ which consists of those homeomorphisms of $\bar{U}$ onto itself which are supported on $U$-that is, which are the identity on $\bar{U}-U$. Then it follows easily that $H^{\prime}$ is a closed subgroup of $G^{\prime}$, and $\bar{U}$ is locally setwise homogeneous under $H^{\prime}$. Thus, by Theorem $2.1, H^{\prime}$ is at least 1-dimensional. 
Let $H(X)$ be the subgroup of $G(X)$ which consists of those homeomorphisms of $X$ onto itself which are supported on $U$. Then $H(X)$ can be identified with $H^{\prime}$, and it follows that $H(X)$, and therefore $G(X)$, is at least 1-dimensional.

Question. Let $X$ be a locally setwise homogeneous continuum. What is the dimension of $G(X)$ ? Must $G(X)$ be infinite dimensional? Can it be of finite positive dimension?

3. Applications of Theorem 2.1. In this chapter we first show that the universal plane curve $\left({ }^{9}\right)$ and the universal curve $\left({ }^{9}\right)$ are each locally setwise homogeneous. Thus it follows, as a corollary of Theorem 2.1, that the groups of all homeomorphisms of these continua are at least 1-dimensional.

We next assert that the group, $H$, of those homeomorphisms of a compact $n$-manifold $M^{n}, n>1$, which carry a countable dense subset of $\left(M^{n}\right)^{0}$ onto itself is at least 1-dimensional. We note in Corollary 2 of Lemma 3.3 that $M^{n}$ is locally setwise homogeneous under $H$. However, by Corollary 1 of Lemma 3.3, $H$ is dense in $G\left(M^{n}\right)$. Thus Theorem 2.1 does not apply directly, since the limit homeomorphism $h$, of the sequence of homeomorphisms $\left\{h_{i}\right\}$ of $H$, may not be in $H$. However, in the latter half of this section, we will indicate how the proof of Theorem 2.1 can be modified so that $h$ must be in $H$.

Definition 3.1. The standard construction for the universal plane curve is the following: Let $S$ be a square plus its interior in the plane. Divide $S$ into 9 equal squares, and remove the interior of the middle ninth. Break each of the remaining 8 squares into 9 equal squares, and remove the interiors of their middle ninths. Continue the process inductively. The set which remains is a continuum called the universal plane curve.

This continuum has been characterized by G. T. Whyburn [3] as the only locally connected, 1-dimensional, plane continuum with no local cut points.

If $M$ is any such continuum imbedded in the plane, then the simple closed curve which bounds the unbounded complementary domain of $M$ is called the outer boundary of $M$ and is denoted by $C_{0}$. The collection of simple closed curves, different from $C_{0}$, and which bound complementary domains of $M$, will be denoted by $\left\{C_{i}\right\}_{i=1}^{\infty}$. The points of $M$ not on any complementary bounding simple closed curve are called its interior points.

DEFINITION 3.2. The standard construction for the universal curve is the following: Let $F_{1}, F_{2}$, and $F_{3}$ be 3 faces of the solid cube $C$ in $E^{3}$ such that no 2 of these faces are opposite each other. We remove portions of $C$ by punching out, to the opposite side, the interiors of the middle ninths of $F_{1}, F_{2}$, and $F_{3}$. We next punch out, to the opposite side, the interiors of the middle ninths of each of the remaining 8 squares in $F_{1}, F_{2}$, and $F_{3}$, and we continue the process inductively. The remaining set is a continuum called the universal curve.

This continuum has been characterized by R. D. Anderson, Theorem 12 of [2],

(9) See below for definition. 
as the only locally connected, 1-dimensional continuum with no local cut points, such that no open subset is imbeddable in the plane.

Definition 3.3. Let $M$ be a separable metric space such that, for some $n$, each point of $M$ has a neighborhood whose closure is homeomorphic to an $n$-cell. Then $M$ is called a manifold.

LEMma 3.1. Let $M$ be the universal plane curve. Then there exists a basis, $\mathscr{E}$, of connected open sets of $M$ such that the closure of each element of $\mathscr{E}$ is homeomorphic to the universal plane curve.

Proof. We think of $M$ as having its standard construction in the plane. First we will decompose $M$ into 4 "equal" subcontinua, which intersect, pairwise, in Cantor sets or in the empty set. We accomplish this by chopping up $M$ by means of a vertical and a horizontal line in $E^{2}$ through the midpoints of 2 adjacent sides. Each of the 4 subcontinua is the closure of an open set and is also a universal plane curve, whose outer boundary meets both the interior of $M$ and also points of some of the complementary bounding simple closed curves of $M$.

We next break up each of the 4 universal plane curves in a similar manner, thus obtaining 16 "equal" universal plane curves, each of which is the closure of an open set, and such that the intersection of any two is empty or is a Cantor set. Continue the process inductively. We observe that the diameters of these sets have limit 0 .

We now enlarge each of these sets slightly, accomplishing this again by means of vertical and horizontal lines near the lines determining these sets, and intersecting $M$ in Cantor sets. Then the collection of interiors of each of these new sets forms the desired basis.

LEMMA 3.2. Let $M$ be a universal plane curve, and let $a$ and $b$ be interior points of $M$. Then there exists an arc $N$ in $M$, such that $N$ contains $a$ and $b$, and $N$ lies in the interior of $M$, except for its endpoints which are in $C_{0}$.

Proof. By a theorem of R. L. Moore [7, p. 363] there exists a monotone map $\phi$ of $M$ onto a disk $D$, (which we think of as the unit disk in $E^{2}$ ), such that the nondegenerate inverses of $M$ are the elements of $\left\{C_{i}\right\}_{i=1}^{\infty}$. Let $C=\bigcup_{i=1}^{\infty} C_{i}$, and let $A=\phi(C)$. Then $A$ is a countable dense subset of the interior of $D$, and $\phi(a)$ and $\phi(b)$ are two points of $D^{0}-A$.

Let $P_{1}$ be a polygonal arc from some point of $\mathrm{Bd} D$ to $\phi(a)$, which misses $A \cup\{\phi(b)\}$ and which lies, except for one endpoint, in $D^{0}$. Let $P_{2}$ be a polygonal arc in $D^{0}$ from $\phi(a)$ to $\phi(b)$ and which misses $A \cup P_{1}^{0}$. Let $P_{3}$ be a polygonal arc from $\phi(b)$ to some point of $\mathrm{Bd} D$ which misses $A \cup P_{1} \cup P_{2}^{0}$ and which lies, except for one endpoint, in $D^{0}$. Thus $P=P_{1} \cup P_{2} \cup P_{3}$ is a polygonal arc which lies, except for its endpoints, in $D^{0}-A$.

Let $N=\phi^{-1}(P)$. Then $N \subset M$, and is the desired arc. 
THEOREM 3.1. The universal plane curve, $M$, is locally setwise homogeneous.

Proof. Let $B$ be the set of interior points of $M$. Then $B$ is a dense subset of $M$. Let $\mathscr{E}$ be the basis of open sets constructed in Lemma 3.1. Let $E \in \mathscr{E}$, and let $a, b \in B \cap E$. We will show that there exists an $h \in G(M)$ such that $h$ is supported on $E$ and $h(a)=b$.

By Lemma 3.2, there exists an arc $N_{0}$ in $\bar{E}$ such that $N_{0}$ contains $a$ and $b$, and $N_{0}$ lies in the interior of $\bar{E}$, except for its endpoints, which are in the outer boundary of $\bar{E}$.

The outer boundary of $\bar{E}$, together with $N_{0}$, forms a $\theta$-curve, $N$. Let $N=N_{1} \cup N_{2}$ where $N_{1}$ and $N_{2}$ are simple closed curves such that $N_{1} \cap N_{2}=N_{0}$, and $N_{1}$ is the outer boundary of the universal plane curve $Q_{1}$, and $N_{2}$ is the outer boundary of the universal plane curve $Q_{2}$.

Let $\phi$ be any homeomorphism of $N$ onto itself such that $\phi$ carries $a$ to $b$ and is the identity on $N-N_{0}$. We note that $\phi$ is the identity on the outer boundary of $\bar{E}$. Let $\phi_{1}=\phi \mid N_{1}$, and let $\phi_{2}=\phi \mid N_{2}$. By Theorem 1 of [3], there exist extensions $\phi_{1}^{*}$ of $\phi_{1}$ to $Q_{1}$ and $\phi_{2}^{*}$ of $\phi_{2}$ to $Q_{2}$. Let $h$ be the homeomorphism of $M$ onto itself which is the identity outside $\bar{E}, \phi_{1}^{*}$ on $Q_{1}$, and $\phi_{2}^{*}$ on $Q_{2}$. Then $h$ is a homeomorphism of $M$ onto itself, $h$ is supported on $E$, and $h(a)=b$. Thus, the universal plane curve is locally setwise homogeneous.

COROLlaRY 3.1.1. The group of all homeomorphisms of the universal plane curve is at least 1-dimensional.

Proof. This follows immediately from Theorem 2.1 and Theorem 3.1 above.

THEOREM 3.2. The universal curve is locally setwise homogeneous.

Proof. This is just a rewording of a statement given in the proof of Theorem 16 of [2].

COROLlARY 3.2.1. The group of all homeomorphisms of the universal curve is at least 1-dimensional.

Proof. This follows immediately from Theorem 2.1 and Theorem 3.2 above.

We next consider the group, $H$, of those homeomorphisms of a compact $n$-manifold, $M^{n}, n>1$, which carry a countable dense subset, $A=\left\{a_{i}\right\}_{i=1}^{\infty}$ of $\left(M^{n}\right)^{0}$ onto itself. We indicate how the proof of Theorem 2.1 can be modified to show that $H$ is at least 1 -dimensional.

Modification of proof of Theorem 2.1. We will establish below, in Lemmas 3.3, 3.4, and 3.5, the existence in $H$ of sliding homeomorphisms of $k$ units, $1 \leqq k \leqq m$, on a chain $\mathscr{C}: C_{1}, \cdots, C_{m}$, in $M^{n}$. Thus we may choose the sequences of sliding homeomorphisms, $\left\{h_{i}\right\}$ and $\left\{g_{i}\right\}$ of Theorem 2.1, to be elements of $H$. Since the manifold is of dimension greater than 1 , we may further require that $\mathscr{C}_{i}^{*}$ miss $a_{i}$. Thus $\bigcap_{i} \mathscr{C}_{i}^{*}$ misses $A$, and the limit homeomorphism, $h$, carries $A$ onto itself. It follows that $h \in H$, and thus $H$ is nonzero dimensional. 
We now prove the necessary lemmas.

LEMMA 3.3. Let $U$ be a connected open subset of a compact manifold, $M$, and let $A=\left\{a_{i}\right\}_{i=1}^{\infty}$ be a countable dense subset of $U \cap M^{0}$. Let $h$ be $a$ homeomorphism supported on $U$. Then for each $\varepsilon>0$, there exists a homeomorphism $g$ supported on $U$ such that $d(h, g)<\varepsilon$ and $g(A)=A$.

Proof $\left({ }^{10}\right)$. Let $\left\{\varepsilon_{i}\right\}$ be a decreasing sequence of positive numbers such that $\sum \varepsilon_{i}<\varepsilon$. Let $b_{1}$ be the first point in the sequence $\left\{a_{i}\right\}$ such that at least one of $h^{-1}\left(b_{1}\right)$ and $h\left(b_{1}\right)$ is not in $A$. Let $U_{1}$ and $V_{1}$ be disjoint $\varepsilon_{1}$-neighborhoods of $b_{1}$ and $h\left(b_{1}\right)$, respectively, whose boundaries miss $A \cup h(A)$, and each of which misses $a_{i}$ and $h\left(a_{i}\right)$ for each $a_{i}$ which precedes $b_{1}$ in the ordering of $A$. Let $\phi_{1}$ be. a homeomorphism supported on $U_{1}$ and carrying some point of $h(A)$ to $b_{1}$, if $h^{-1}\left(b_{1}\right)$ is not in $A$. Otherwise, let $\phi_{1}$ be the identity. Let $\psi_{1}$ be a homeomorphism supported on $V_{1}$ and carrying $h\left(b_{1}\right)$ to some point of $A$, if $h\left(b_{1}\right)$ is not in $A$. Otherwise, let $\psi_{1}$ be the identity. Let $g_{1}=\psi_{1} \phi_{1} h$. Then $d\left(h, g_{1}\right)<\varepsilon_{1}$, and $g_{1}^{-1}\left(b_{1}\right)$ and $g_{1}\left(b_{1}\right)$ are in $A$ as well as $g_{1}^{-1}\left(a_{i}\right)$ and $g_{1}\left(a_{i}\right)$ for each $a_{i}$ preceeding $b_{1}$. The homeomorphism $g_{1}$ is a first approximation to $g$.

We again note that $g_{1}^{-1}\left(a_{i}\right)$ and $g_{1}\left(a_{i}\right)$ are elements of $A$ for all $a_{i}$ which precede $b_{1}$, as well as for $b_{1}$. Let $b_{2}$ be the first point in the ordering of $A$ such that at least one of $g_{1}^{-1}\left(b_{2}\right)$ and $g_{1}\left(b_{2}\right)$ is not in $A$. Since $g_{1}^{-1}$ is uniformly continuous, there is a $\delta_{1}>0$ such that $g_{1}^{-1}$ takes any $\delta_{1}$-set to a set of diameter $<\varepsilon_{2}$. Let $U_{2}$ and $V_{2}$ be two disjoint, connected, open sets of diameter less than $\min \left\{\delta_{1}, \varepsilon_{2}\right\}$ and containing $b_{2}$ and $g_{1}\left(b_{2}\right)$ respectively, with the following restrictions:

(1) if $b_{2} \in U_{1}$ (or $V_{1}$ ) then $\bar{U}_{2} \subset U_{1}$ (or $V_{1}$ ),

(2) if $g_{1}\left(b_{2}\right) \in U_{1}$ (or $\left.V_{1}\right)$ then $\bar{V}_{2} \subset U_{1}$ (or $V_{1}$ ),

(3) if $b_{2} \in C\left(U_{1} \cup V_{1}\right)$ then $\bar{U}_{2} \subset C\left(U_{1} \cup V_{1}\right)$, and

(4) if $g_{1}\left(b_{2}\right) \in C\left(U_{1} \cup V_{1}\right)$ then $\bar{V}_{2} \subset C\left(U_{1} \cup V_{1}\right)$.

We also require that $\mathrm{Bd} U_{2}$ and $\mathrm{Bd} V_{2}$ miss $A \cup g_{1}(A)$ (a countable set) and that $U_{2}$ and $V_{2}$ each miss all the (finitely many) points $a_{i}$ and $h\left(a_{i}\right)$ for $a_{i}$ preceding $b_{2}$. Let $\phi_{2}$ be a homeomorphism supported on $U_{2}$ and carrying some point of $g_{1}(A)$ to $b_{2}$, if $g_{1}^{-1}\left(b_{2}\right)$ is not in $A$; let $\psi_{2}$ be a homeomorphism supported on $V_{2}$ and carrying $g_{1}\left(b_{2}\right)$ to some point of $A$, if $g_{1}\left(b_{2}\right)$ is not in $A$. Let $g_{2}=\psi_{1} \phi_{1} g_{1}$. Then $d\left(g_{1}, g_{2}\right)<\varepsilon_{2}$, and $g_{2}^{-1}\left(b_{2}\right)$ and $g_{2}\left(b_{2}\right)$ are in $A$, as well as $g_{2}^{-1}\left(a_{i}\right)$ and $g_{2}\left(a_{i}\right)$ for all $a_{i}$ preceding $b_{2}$.

We continue the above process inductively, with the following additional restriction: we require that $\bar{U}_{n}$ and $\bar{V}_{n}$ be entirely contained in any $U_{i}$ or $V_{i}, i<n$, which it meets. Thus we obtain a sequence of homeomorphisms, $\left\{g_{i}\right\}$, such that $d\left(g_{i-1}, g_{i}\right)<\varepsilon_{i}$. Since $\sum \varepsilon_{i}<\infty,\left\{g_{i}\right\}$ is Cauchy, and thus converges to a map $g$. We will show that $g$ is $1-1$, and therefore a homeomorphism.

Let $x \neq y$, and let $\delta=d(x, y)$. There exists an integer $N$ such that for $n>N$,

(10) This proof was outlined to me by R. D. Anderson. 
the diameter of $g_{n-1}^{-1}\left(U_{n}\right)<\delta$ and the diameter of $g_{n-1}^{-1}\left(V_{n}\right)<\delta$. Then not both $g_{n-1}(x)$ and $g_{n-1}(y)$ can be in the same one of these sets $U_{n}$ or $V_{n}$. Thus if $g_{n-1}(x) \in U_{n}$ (or $\left.V_{n}\right)$ and $g_{n-1}(y) \notin U_{n}$ (or $V_{n}$ ), then because of the (partial)! towering of the $U_{i}$ 's (or $V_{i}$ 's), $g(x) \in U_{n}$ (or $V_{n}$ ) and $g(y) \notin U_{n}$ (or $V_{n}$ ); that is, $g(x) \neq g(y)$. If neither $g_{n-1}(x)$ nor $g_{n-1}(y)$ is in any $U_{n}$ or $V_{n}$ for $n>N$, then each is determined at a finite stage; that is, $g(x)=g_{N}(x)$ and $g(y)=g_{N}(y)$ for some $N$, and since $g_{N}$ is a homeomorphism, $g_{N}(x) \neq g_{N}(y)$. So again we have $g(x) \neq g(y)$. Thus $g$ is $1-1$ and therefore a homeomorphism.

Since $d\left(h, g_{1}\right)<\varepsilon_{1}$, and $d\left(g_{i-1}, g_{i}\right)<\varepsilon_{i}$, it follows that $d(h, g)<\sum \varepsilon_{i}<\varepsilon$. Thus $g$ is the desired homeomorphism.

REMARK. In the above proof, the sequence $\left\{\varepsilon_{i}\right\}$ could have been chosen to be a decreasing sequence of numbers with $\varepsilon_{1}<\varepsilon$, and so that $\varepsilon_{i} \rightarrow 0$. The limit map $g$ would still exist and be the desired homeomorphism.

COROLLARY 3.3.1. The group of those homeomorphisms of a compact manifold, $M$, taking a countable dense subset of $M^{0}$ onto itself, is dense in the group of all homeomorphisms of the manifold.

Proof. Clear.

Corollary 3.3.2. Let $M$ be a compact manifold, $A$ be a countable dense subset of $M^{0}$, and $H$ be the group of those homeomorphisms of $M$ onto itself, which carry $A$ onto itself. Then $M$ is locally setwise homogeneous under $H$.

Proof. Let $U$ be a connected open set in $M$, let $a, b \in U \cap A$. It is easy to show that there is a homeomorphism $h \in G(M)$ such that $h(a)=b$, and $h$ is supported on $U$. By Lemma 3.3, $h$ can be modified to a homeomorphism $g$ such that $g$ is supported on $U$ and $g(A)=A$. We can insure that $g(a)=b$, simply by choosing each of the $U_{i}$ 's and $V_{i}$ 's of Lemma 3.3 so that they miss the point $b$.

Thus $g$ is a homeomorphism supported on $U$ such that $g(a)=b, g(A)=A$, and $g \in H$. It follows that $M$ is locally setwise homogeneous under $H$.

Remark. We observe that if $M$ is a 1 -manifold, then $H$ is zero dimensional. (This is easy to prove.) Thus local setwise homogeneity of a space under a group, $H$, is not sufficient to guarantee that $H$ is at least 1-dimensional. Therefore the hypothesis of Theorem 2.1, that $H$ be closed, cannot be omitted without substituting some other condition to insure that $H$ is at least 1-dimensional.

LEMMA 3.4. Let $\mathscr{C}: C_{1}, \cdots, C_{n}$ be a chain of connected open spheres of the compact manifold, $M$, whose boundaries miss a countable dense set $A$ of $M^{0}$. Let $h$ be a sliding homeomorphism of $k$ units on $\mathscr{C}$, let $\delta=\min _{i}\left\{d\left(\bar{C}_{i-2}, h\left(\bar{C}_{i}\right)\right)\right\}$, and let $\varepsilon<\delta$. We note that $\delta>0$. Then there exists a homeomorphism $g$ such that $d(g, h)<\varepsilon, g(A)=A$, and $g$ is also a sliding homeomorphism of $k$ units on $\mathscr{C}$.

Proof. Let $\mathscr{D}: D_{1}, \cdots, D_{n-k}$ be a chain so that (1) $D_{i} \subset C_{i+k}$ and $(2) h^{-1}\left(D_{i}\right) \subset C_{i}$. 
We may further require that $\bigcup_{i}\left\{\mathrm{Bd} D_{i}\right\}$ miss $A \cup h(A)$, for, if not, we can always choose a chain $\mathscr{E}$ which is a straight refinement of $\mathscr{D}$ and such that the boundary of each of its links misses $A \cup h(A)$. Then the chain whose elements are $\left\{\mathscr{E}_{i}^{*}\right\}_{i=1}^{n-k}$ satisfies all these properties.

We construct $g$ as a limit of a sequence $\left\{g_{i}\right\}$, as in Lemma 3.3, with the following additional restriction: each $\bar{U}_{i}$ and $\bar{V}_{i}$ is a subset of either $\mathscr{D}^{*}$ or $C\left(\mathscr{D}^{*}\right)$, and lies entirely in any $C_{j}$ which it meets. Then $d(g, h)<\varepsilon, g(A)=A$, and $g$ is also a sliding homeomorphism of $k$ units on $\mathscr{C}$.

LemMA 3.5. Let $M$ be a manifold, let $\mathscr{C}: C_{1}, \cdots, C_{n}$ be a chain of connected open sets of $M$, and let $A$ be a countable dense subset of $M^{0}$. Let $H$ be the group of those homeomorphisms of $M$ which carry $A$ onto itself. Then for each integer $k, 1 \leqq k \leqq n$, there exists a sliding homeomorphism, $h$, of $k$ units on $\mathscr{C}$, and such that $h \in H$.

Proof. This follows from Lemma 3.4 above, and Lemma 2.4 of §2, which asserts the existence of sliding homeomorphisms.

Thus, with the proofs of these lemmas, we have completed (the outline of) the proof of the following:

THEOREM 3.3. The group of those homeomorphisms of a compact n-manifold, $M^{n}, n>1$, which carry a countable dense subset of $\left(M^{n}\right)^{0}$ onto itself, is at least 1-dimensional.

QuEstion 1. What is the dimension of the group of homeomorphisms of the universal plane curve? universal curve?

QUESTION 2. What is the dimension of the group of those homeomorphisms of a compact $n$-manifold, $n>1$, which carry a countable dense subset of the interior of the $n$-manifold onto itself?

4. 1-dimensional continua. In this section we investigate the dimensions of the groups of homeomorphisms of 1-dimensional continua. It is known and easy to show (see Theorem 1.1) that the group of homeomorphisms of an arc is infinite dimensional. On the other hand, there are dendrons and other 1-dimensional continua [8] which admit no homeomorphisms onto themselves, other than the identity.

Our main results are the following:

(1) The group of homeomorphisms, $G$, of a regular curve $\left({ }^{11}\right), M$, is either 0 -dimensional or infinite dimensional. $G$ is infinite dimensional if $M$ contains a free $\operatorname{arc}\left({ }^{12}\right)$, 0-dimensional otherwise.

(11) A regular curve is a continuum in which every point has arbitrarily small neighborhoods with finite boundaries $[5$, p. 96]. A regular curve is locally connected $[4$, p. 99].

(12) A free arc is an arc whose interior is an open subset of the space. 
(2) For each positive integer $n$, there exists a rational curve $\left({ }^{13}\right), M_{n}$, such that $1 \leqq \operatorname{dim} G\left(M_{n}\right) \leqq n$, and $G\left(M_{n}\right)$ is homeomorphic to the product of $n$ 1-dimensional groups.

Definition 4.1. Let $M$ be a regular curve, $B$ an order basis $\left({ }^{14}\right)$ for $M$. Let $\mathscr{W}: W_{1}, \cdots, W_{n}$ be a cover of $M$ of mesh $<\varepsilon$ with the following properties for each $i$ :

(1) $W_{i}$ is a continuum which is the closure of an open set,

(2) $\mathrm{Bd} W_{i}$ is a finite subset of $B$, and

(3) $W_{i} \cap W_{j}=\operatorname{Bd} W_{i} \cap \operatorname{Bd} W_{j}$ for each $j \neq i$. Then $\mathscr{W}$ is called a regular $\varepsilon$-cover with respect to $B$.

Lemma 4.1. Let $M$ be a regular curve and let $B$ be an order basis for $M$ which contains all the local cut points of $\operatorname{order}\left({ }^{15}\right) \geqq 3$. Then for each $\varepsilon>0$, there exists a regular $\varepsilon$-cover with respect to $B$.

Proof. Let $\mathscr{U}$ be a finite $\varepsilon / 3$-cover of $M$ whose elements have finite boundaries. We will modify the cover $\mathscr{U}$ to obtain a cover $\mathscr{U}^{\prime}$, each of whose elements has a finite boundary in $B$. Let $B^{\prime}$ be the set of boundary points of elements of $\mathscr{U}$. This is a finite set, and therefore there exists a $\delta>0$ such that $d\left(b_{i}, b_{j}\right)>\delta$ for each pair $b_{i}, b_{j} \in B^{\prime}$. Let $b \in B^{\prime}$ on the boundary of some $U \in \mathscr{U}$. If $b$ is also in $B$, we leave it alone. If not, there are 2 possibilities: (1) $b$ is not a local cut point, or (2) $b$ is a local cut point of order 2 . In the first case $U \cup\{b\}$ is also open, and we add $b$ to $U$. In the second case, there exists a neighborhood $N_{b}$ of $b$ of diameter $<\min \{\delta, \varepsilon / 3\}$ with $\mathrm{Bd} N_{b}$ containing at most 2 points in $B$, since $B$ is an order basis for $M$. We add such a neighborhood to $U$. Thus we have modified each element of $\mathscr{U}$ a finite number of times. We now have a new cover $\mathscr{U}^{\prime \prime}$ of open sets of diameter $<\varepsilon$, each of which has a finite boundary which is a subset of $B$. We choose a minimal subcover $\mathscr{U}^{\prime}$ of $\mathscr{U}^{\prime \prime}$.

Let $\mathscr{U}^{\prime}$ have the elements $U_{1}^{\prime}, U_{2}^{\prime}, \cdots, U_{m}^{\prime}$. Then we obtain a new cover $\mathscr{V}$ in the following way: let

$$
V_{1}=\overline{U_{1}^{\prime}}, \quad \overline{V_{k}=U_{k}^{\prime}-\bigcup_{i=1}^{k-1} \overline{U_{i}^{\prime}}} \text { for } k>1 .
$$

Then $\mathscr{V}$ has at most $m$ elements. $\mathscr{V}$ is a finite cover of $M$ of mesh $<\varepsilon$ whose elements are the closures of open sets, and whose boundaries are finite subsets of $B$. Let $\mathscr{W}$ be the collection of components of the elements of $\mathscr{V}$. Since each element

(13) A rational curve is a continuum in which every point has arbitrarily small neighborhoods with countable boundaries [5, p. 96]. A rational curve need not be locally connected.

(14) $B$ is an order basis for a regular curve $M$ if every point $x \in M$ has arbitrarily small neighborhoods $U_{x}$ with boundaries in $B$ such that the number of points on $\operatorname{Bd} U_{x}$ is less than or equal to the order of $x[5$, p. 277]. (See footnote 15 for definition of "order".)

(15) The order of a point $x$ of $M$ is the smallest number $n$ for which there exist arbitrarily small neighborhoods with boundaries consisting of less than or equal to $n$ points, if such exists. If not, we say the point is of infinite order [5, p. 97-99]. 
of $\mathscr{V}$ has only a finite number of boundry points, and each of these can belong to at most 1 component of that element, $\mathscr{W}$ is finite. Then $\mathscr{W}$ is the desired cover.

Lemma 4.2. Let $M$ be a regular curve, $b$ a local cut point of $M$, and $N a$ connected neighborhood of $b$ such that $N-b=A_{1} \cup A_{2}$ where $A_{1}$ and $A_{2}$ are mutually separate sets. Let $S$ be a sphere about $b$ (the set of all points $a$ fixed distance from $b$ ) such that $S \subset N$ and $S \cap A_{1} \neq \varnothing$ and $S \cap A_{2} \neq \varnothing$. Then there exists an arc $C$ from some point of $S \cap A_{1}$ to some point of $S \cap A_{2}$ such that $C^{0}$ is a subset of the "interior" of $S$.

Proof. Standard.

LEMMA 4.3. Let $M$ be a regular curve, $U$ be a connected open subset of $M$ such that $\bar{U}$ contains no local cut points of $M$ of order $>2$ and such that $\mathrm{Bd} U$ is a finite set of local cut points of $M$. Then $\bar{U}$ is a regular curve containing no local cut points of order $>2$ (in $\bar{U})$.

Proof. Clear.

LEMMA 4.4. Let $M$ be a regular curve containing no local cut points of order $>2$. Then $M$ is an arc or a simple closed curve.

Proof. It is sufficient to show that every point of $M$ is of order at most two [5, p. 267]. Let $B$ be a countable dense set of local cut points of order two of $M$. We know that $B$ exists by $[4$, p. 63]. Then $B$ is an order basis for $M[5$, p. 278].

Suppose there exists a point $p^{\prime} \in M$ of order greater than two. We show that there then exists a point $p \in M$ of order greater than two such that some arc of $M$ contains $p$ in its interior. Let $W$ be a neighborhood of $p^{\prime}$ such that $\bar{W}$ is connected, Bd $W$ contains at least three points, and the boundary of each small regular neighborhood of a boundary point of $W$ contains one point in $C(W)$. Let $a_{1}$ and $a_{2}$ be two points of Bd $W$. Let $A_{1}$ be an arc from $a_{1}$ to $p^{\prime}$ in $\bar{W}$, missing $a_{2} . A_{1}$ exists since the boundary of a sufficiently small regular neighborhood of $a_{2}$ must have a point in $C(W)$, but if $A_{1}$ contained $a_{2}$ in its interior, both boundary points of small regular neighborhoods of $a_{2}$ would be in $W$. Similarly, there exists an arc $A_{2}$ from $a_{2}$ to $p^{\prime}$ in $W$ missing $a_{1}$. Let $p^{\prime \prime}$ be the first point of $A_{2}$ in the order from $a_{2}$ to $p^{\prime}$, which is also on $A_{1}$. Then $p^{\prime \prime} \neq a_{1}$. If $p^{\prime \prime}=p^{\prime}$, then let $p=p^{\prime}$, and $A_{1} \cup A_{2}$ is the required arc. If $p^{\prime \prime}$ precedes $p^{\prime}$ on $A_{1}$, then $p^{\prime \prime}$ is a point of order at least three. Let $p=p^{\prime \prime}$, and $A_{1}$ is the required arc.

Thus we may suppose, by way of contradiction, that there exists a point $p$ of order greater than two such that some arc $S$ of $M$ contains $p$ in its interior. Let $U$ be a small regular neighborhood of $p$ such that $\bar{U}$ is connected, and the boundary of each small regular neighborhood of a boundary point of $U$ must have one of its elements in $C(U)$. Let $a$ be a boundary point of $U$ on $S$, and let $A$ be an arc from $a$ to $p$ in $\bar{U}$ such that $A \subset S$. Then $A$ must contain local cut points of $\bar{U}$ and 
therefore of $M$. For let $V$ be any sufficiently small regular neighborhood of $a$. Then Bd $V$ consists of two points, one of which (say $q$ ), is in $U$. Therefore $q$ will separate $a$ from $p$ in $\bar{U}$, and therefore $q$ will be a local cut point of $M$.

Now, let $N$ be the set of local cut points of $M$ on $A$ which separate $a$ from $p$ in $\bar{U}$, and let $b$ be the lub of $N$ in the order from $a$ to $p$. We will show that $b \neq p$ and $b=p$ each lead to a contradiction. Thus, our original assumption that there is a point of order greater than two in $M$ must be false.

Case 1. Suppose $b=p$. Let $A^{\prime}$ be the arc which is the union of the following two arcs: $A$ and the $\operatorname{arc} A^{\prime \prime}$ from $p$ to some point $c$ on $S$ such that

(1) $p$ is between $a$ and $c$, and

(2) the arc from $p$ to $c$ on $S$ is a subset of $U$.

There exists a sequence of points $\left\{x_{i}\right\}$ converging to $p$ on $A^{\prime}$ such that $x_{i}$ separates $a$ from $p$ in $\bar{U}$. We show that $x_{i}$ separates $a$ from $c$ in $\bar{U}$. If there exists an arc $T$ in $\bar{U}$ from $a$ to $c$ missing $x_{i}$, then $T$ together with the arc from $p$ to $c$ on $S$ is a connected set containing $a$ and $p$. Thus $x_{i}$ would not separate $a$ from $p$. This is a contradiction. Therefore $x_{i}$ must separate $a$ from $c$ in $\bar{U}$.

Now $p$ is the lub $\left\{x_{i}\right\}$, and $p$ precedes $c$. We show that $p$ also separates $a$ from $c$ in $\bar{U}$. Suppose not. Then there exists an arc $Y$ from $a$ to $c$ in $\bar{U}$ missing $p$. Let $y$ be the last point of $Y \cap A$ in the order from $a$ to $p$. Then there exists an integer $n$ such that $x_{n}$ is between $y$ and $p$. Further $x_{n}$ is not on $Y$. Thus the portion of $Y$ from $y$ to $c$, together with the portion of $A$ from $a$ to $y$ is a connected set containing $a$ and $c$, but missing $x_{n}$. Thus $x_{n}$ does not separate $a$ from $c$. This is a contradiction. It follows that $p$ separates $a$ from $c$ in $\bar{U}$.

Therefore $p$ is a local cut point of $M$. But then $p$ must be of order two. But again, this is a contradiction, since our original assumption was that $p$ was of order at least three. Thus the assumption that $b=p$ is false.

Case 2. Suppose $b \neq p$. Then $b$ precedes $p$ on $A$; that is, $b$ is between $a$ and $p$. If $b \in N$, then $b$ is of order two. Otherwise, there exists a sequence $\left\{y_{i}\right\}$ of points on $A$ such that (1) $y_{i} \rightarrow b$ with $y_{i}$ between $a$ and $b$ and (2) $y_{i}$ separates $a$ from $p$ in $\bar{U}$. By an argument similar to that in Case $1, b$ also separates $a$ from $p$ in $\bar{U}$. Thus $b$ must be a local cut point of $M$ and therefore must be of order two. Let $O$ be a small regular neighborhood of $b$, missing $p$, and such that one of the two boundary points of $O$, say $r$, is on $A$ between $b$ and $p$, and the other is on $A$ between $a$ and $b$.

We show that $r$ also separates $a$ from $p$ in $\bar{U}$. Let $D$ be the component of $(\bar{U}-b)$ which contains $a$. Then $D$ is open in $\bar{U}$. Therefore $D \cup O$ is open in $\bar{U}$, contains $a$, misses $p$, and has $r$ as its only boundary point. Thus : $r$ separates $a$ from $p$ in $\bar{U}$.

But this contradicts the fact that $b$ was the lub of $N$. Thus the assumption that $b \neq p$ is false.

Therefore we see that $b=p$ and $b \neq p$ each lead to a contradiction. Thus our very first assumption that there is a point of order at least three in $M$ must be false. 
Thus every point of $M$ is of order at most two. The points of $B$ are of order two. Therefore $M$ is a curve of the second order $\left({ }^{16}\right)$, and it follows that $M$ is an arc or a simple closed curve [5, p. 267].

THEOREM 4.1. Let $M$ be a regular curve with a dense set of local cut points of order $>2$. Then $G(M)$ is 0-dimensional.

Proof. It is sufficient to show that for each $\varepsilon>0$, there is an open and closed set $H$ of diameter $<4 \varepsilon$ which contains the identity.

Let $\varepsilon>0$, and let $B$ be the set of local cut points of order $>2$ of $M$. We know that $B$ is countable and dense and therefore an order basis for $M[5$, pp. 164, 278]. By Lemma 4.1, there exists a cover $\mathscr{W}$ of $M$ which is a regular $\varepsilon$-cover with respect to $B$.

Let $B^{\prime}=\left\{b \in B\right.$ : there exist $i, j, i \neq j$, with $b$ a point of $\left.W_{i} \cap W_{j}, W_{i}, W_{j} \in \mathscr{W}\right\}$. Since $M$ is locally connected, and $B^{\prime}$ is a finite set, we can find about each $b \in B^{\prime}$ a locally connected neighborhood $N_{b}$ such that the set $\left\{N_{b}: b \in B^{\prime}\right\}$ satisfies the following property: $\bar{N}_{b_{i}} \cap \bar{N}_{b_{j}}=\varnothing$, if $b_{i} \neq b_{j}$. Let $S_{b}$ be a sphere about $b$ (the set of all points a fixed distance from $b$ ) which is contained in $N_{b}$ and which contains no point of $B$. We further require that $S_{b}$ be small enough so that it contains points of each $W_{i}$ which contains $b$. Such a sphere exists, since $B$ is countable. Let $S=\bigcup_{b \in B^{\prime}} S_{b}$, and let $A_{i}=S \cap W_{i}$. We note that $A_{i}$ is a closed subset of $W_{i}^{0}$.

Let $H=\left\{h \in G(M): h\left(A_{i}\right) \subset W_{i}\right\}$. It is clear that $e \in H$, since $e\left(A_{i}\right)=A_{i} \subset W_{i}$. We show that $H$ is open, closed, and of diameter $<4 \varepsilon$.

We first show that $H$ is open. Let $h \in H$. Then $h\left(A_{i}\right)$ is a closed subset of $W_{i}^{0}$. Let $\delta=\min _{i}\left\{d\left(h\left(A_{i}\right)\right.\right.$, complement of $\left.\left.W_{i}^{0}\right)\right\}$. Since these are disjoint closed sets, $\delta$ is positive. Let $K$ be a $\delta$-sphere of homeomorphisms about $h$. We show that $K \subset H$. Let $g \in K$. Then $d(h(x), g(x))<\delta$ for all $x$, in particular for $x \in A_{j}$. Therefore $g\left(A_{j}\right) \subset W_{j}$ and it follows that $g \in H$.

We next show that $H$ is closed. Let $\left\{h_{n}\right\}$ be a sequence of homeomorphisms of $H$ such that $\left\{h_{n}\right\}$ converges to some homeomorphism $h$. We show that $h \in H$. Let $x \in A_{k}$. Then $h_{n}(x) \in W_{k}$ for all $n$. Since $W_{k}$ is closed, $h(x) \in W_{k}$ also. Therefore $h\left(A_{k}\right) \subset W_{k}$; that is, $h \in H$.

Finally we show that the diameter of $H$ is less than $4 \varepsilon$. Clearly $e \in H$. It is sufficient to show that if $h \in H, d(h, e)<2 \varepsilon$. It then follows by the triangle inequality that diameter $H<4 \varepsilon$. To this end, let $h \in H$ and suppose that $x \in W_{i_{1}}$ and $h(x) \in W_{i_{2}}$ where $W_{i_{1}} \cap W_{i_{2}}=\phi$. We will show that this is impossible; that is, $d(x,(h(x))<2 \varepsilon$.

Let $B^{\prime \prime}=\left\{b_{1}, \cdots, b_{r}\right\}$ be the points of $\mathrm{Bd} W_{i_{2}}$; that is, the points of $B^{\prime} \cap W_{i_{2}}$. Let $C_{j}$ be an arc from some point of $A_{i_{2}}$ to some point of $A_{i}$ for $i \neq i_{2}$, such that $C_{j} \subset\left(S_{b_{j}}\right.$ plus its interior). By Lemma 4.2 , such $C_{j}$ 's exist. We note that $C_{j}$ contains $b_{j}$. We therefore have a finite collection of pairwise disjoint arcs $C_{1}, \cdots, C_{r}$ whose union contains $B^{\prime \prime}$. Since each of these arcs $C_{i}$ has an endpoint whose image in is

(16) A curve of the nth order is a continuum which contains no points of order $>n$, but at least 1 point of order $n[5$, p. 98]. 
$W_{i_{2}}^{0}$ and an endpoint whose image is not in $W_{i_{2}}$, and since $B^{\prime \prime}$ separates $W_{i_{2}}^{0}$ from $C\left(W_{i_{2}}\right), h\left(C_{i}^{0}\right)$ must contain at least 1 point of $B^{\prime \prime}$. But we have a total of $r$ disjoint arcs (and therefore their images are disjoint) and these must contain at least $r$ points of $B^{\prime \prime}$. Therefore $h\left(\bigcup_{i=1}^{r} C_{i}^{0}\right)$ contains $B^{\prime \prime}$.

We will construct arc $C^{\prime}$ which misses $\bigcup_{i=1}^{r} C_{i}^{0}$, but such that $h\left(C^{\prime}\right)$ must also contain a point of $B^{\prime \prime}$. Let $y \in W_{i_{2}}$. Since $M$ is locally connected, there exists an $\operatorname{arc} C$ from $x$ to $y$. (Recall that $x \in W_{i_{1}} \ni h(x) \in W_{i_{2}}$.) Let $S^{\prime}=\left[\left(\bigcup_{i=1}^{r} S_{b_{i}}\right) \cap C\left(W_{i_{2}}\right)\right]$. Since $B^{\prime \prime}$ separates $W_{i_{2}}{ }^{0}$ from $C\left(W_{i_{2}}\right), S^{\prime}$ separates $W_{i_{2}}$ from all elements of $\mathscr{W}$ not meeting $W_{i_{2}}$. Therefore $C$ must contain a point of $S^{\prime}$. Let $z$ be the first point of $S^{\prime}$ on $C$, and let $C^{\prime}$ be the subarc of $C$ from $x$ to $z$. Then $C^{\prime} \cap\left(\bigcup_{i=1}^{r} C_{i}^{0}\right)=\varnothing$, $h(z) \in W_{j}$ for some $j \neq i_{2}$, and $h(x) \in W_{i_{2}}$. But the last 2 conditions imply that $h\left(C^{\prime}\right)$ must contain some point of $B^{\prime \prime}$. However, as noted above, $B^{\prime \prime} \subset h\left(\bigcup_{i=1}^{r} C_{i}^{0}\right)$ and $C^{\prime} \cap \bigcup_{i=1}^{r} C_{i}^{0}=\varnothing$, and therefore $h\left(C^{\prime}\right) \cap h\left(\bigcup_{i=1}^{r} C_{i}^{0}\right)=\varnothing$. This is a contradiction.

THEOREM 4.2. The group of homeomorphisms of a regular curve $M$ is either 0 - or $\infty$-dimensional. It is $\infty$-dimensional if $M$ contains a free arc. It is 0 dimensional otherwise.

Proof. If $M$ contains a free arc $A$, let $H$ be the set of homeomorphisms of $M$ onto itself, supported on $A$. Then $H$ can be identified with the group of homeomorphisms of an arc keeping the endpoints fixed. But this is $\infty$-dimensional. (See Theorem 1.1.)

If $M$ does not contain a free arc, we show that $M$ must contain a dense set of local cut points of order $>2$, and therefore by Theorem 4.1, $G(M)$ is 0-dimensional. We do this by proving the contrapositive.

Suppose $M$ does not contain a dense set of local cut points of order $>2$. Then there exists a connected, open set $U \subset M$ such that $\bar{U}$ contains no local cut point of $M$ of order $>2$, and such that Bd $U$ is a finite set of local cut points of $M$. (We choose $U$ so that Bd $U$ is contained in some order basis.) By Lemma 4.3, $\bar{U}$ is a regular curve containing no local cut points of order $>2$. By Lemma 4.4, $\bar{U}$ is an arc or a simple closed curve. It follows that $M$ contains a free arc.

Thus we have shown that if $M$ does not contain a free arc, it contains a dense set of local cut points of order $>2$, and thus $G(M)$ is 0-dimensional.

THEOREM 4.3. There exist rational curves whose full groups of homeomorphisms are exactly 1-dimensional.

Discussion of proof. We will construct such continua in more detail in $\S 5$. The properties of their groups of homeomorphisms will be proved directly, or will be similar to, or follow from, other theorems in that section. Briefly, the continua are constructed by use of a sequence of dendrons $\left\{D_{i}\right\}$ converging to a limit arc $D$, in such a way that exactly 1 endpoint of each $D_{i}$ meets the left-hand endpoint of $D$, and exactly 1 other endpoint of each $D_{i}$ meets the right-hand 
endpoint of $D$. This is constructed carefully so that each $D_{i}$ admits only countably many homeomorphisms onto itself, and any homeomorphism of such a continuum onto itself, when restricted to the limit arc $D$, is just a "translation" of $D$ onto itself.

THEOREM 4.4. For each positive integer $n$, there exist rational curves, $R_{n}$, whose full groups of homeomorphisms, $G_{n}$, are at least one and at most n-dimensional. In fact, $G_{n}$ is homeomorphic to the product of $n 1$-dimensional sets.

Discussion of proof. We construct these continua in detail in $\S 5$ also. They are obtained by taking the union, $M$, of $n$ different continua, $M_{i}$, constructed for Theorem 4.3 , in such a way that the $i$ th and $(i+1)$ th meet only at an endpoint of their limit arcs. Then the group of homeomorphisms of $M$ is just the product of the groups of homeomorphisms of the $M_{i}$ 's.

REMARK: If a 1-dimensional continuum is not a rational curve, then its group of homeomorphisms may be 0-dimensional, as for example in [8] or it may be at least 1-dimensional, as in the case of the universal plane curve and the universal curve. Certainly it may be infinite dimensional; simply attach a free arc to a point of the outer boundary of a universal plane curve.

QUESTION 1. Do there exist 1-dimensional nonrational curves whose groups of homeomorphisms are of finite positive dimension?

QUESTION 2. Do there exist one-dimensional, locally connected continua whose groups of homeomorphisms are of finite positive dimension?

5. Continua with finite, positive dimensional groups of homeomorphisms. In this section we will construct continua such that the groups of all homeomorphisms of these continua are exactly 1-dimensional. We then modify these continua by taking, in some cases unions, in other cases sets which are like unions, of these continua, and thus obtain continua, $M_{n}$, such that $1 \leqq \operatorname{dim} G\left(M_{n}\right) \leqq n$, and $G\left(M_{n}\right)$ is the product of $n 1$-dimensional sets. We also show that these continua may be rational curves.

Our method of procedure is the following. We will first construct an auxiliary space-a compact set, $K$, such that $G(K)$ is exactly 1-dimensional. We then "extend" $K$ to a continuum, $M$, such that $G(M)$ is both topologically and algebraically the same as $G(K)$. Thus $M$ is a continuum such that $G(M)$ is exactly 1-dimensional.

Construction of certain dendrons. It is well known that there exist dendrons which admit no homeomorphisms onto themselves other than the identity. Any dendron which is constructed so that it contains a dense set of branch points, no two of which have the same order, is such an example.

We show that there are uncountably many such dendrons. Let $f$ be a function from the set of natural numbers greater than or equal to 3 to the set $\{0,1\}$. Let $D_{f}$ be a dendron which includes exactly 1 cut point of order $n$ iff $f(n)=1$. Since 
there are uncountably many such functions, there are uncountably many such dendrons.

Construction of the continua $K_{n}$. It is easy to show, using the dendrons of the previous section, that for each positive integer $n$, there is a continuum, (in fact, a regular curve $\left({ }^{17}\right), K_{n}$, such that $G\left(K_{n}\right)$ is cyclic of order $n\left({ }^{18}\right)$. We construct these continua for use in the next construction.

Let $C$ be a circle of radius $r$ about the origin in $E^{2}$, and consider the set of points of $C$ with polar coordinates $(r, 2 \pi k / n)$, for each integer $k, 0 \leqq k<n$. These will be called the "vertices" of $K_{n}$. Let $D$ be a dendron admitting exactly one homeomorphism such that

(1) the branch points of $D$ form a dense set of points, no two of which have the same order,

(2) $D$ lies in a neighborhood of the arc $A$ of $C$ from $(r, 0)$ to $(r, 2 \pi / n)$ and contains this arc, $A$,

(3) the endpoints of $A$ are endpoints of $D$,

(4) if $P$ is a point of $D$, other than the endpoints of $A$, and the polar coordinates of $P$ are $(r, \theta)$, then $0<\theta<2 \pi / n$. Let $r_{n}$ be the counterclockwise, period $n$ rotation, about the origin, of $E^{2}$ onto itself. We note that $\left[r_{n}(D)\right] \cap D=(r, 2 \pi / n)$. We define $K_{n}$ as follows: Let $K_{n}=\bigcup_{i=0}^{n-1} r_{n}^{i}(D)$.

Clearly, $G\left(K_{n}\right)$ contains a cyclic group of order $n$. Furthermore these "rotations" are the only homeomorphisms of $K_{n}$ onto itself.

THEOREM (SEE THEOREM 2.3 AND 2.6). There exists a compact set $K$ such that $G(K)$ is exactly 1-dimensional.

Construction of $K$. Let $C_{n}$ be a circle of radius $1-1 / n$ about the origin in $E^{2}$. Then $C_{n} \rightarrow T$, where $T$ is the unit circle about the origin. We construct, in a small neighborhood of each $C_{n}$, a regular curve, $K_{n}$, as constructed above, so that $K_{n} \cap K_{m}=\phi$, for $n \neq m$. Since, for our construction, $K_{m} \supset C_{m}$, for all $m$, in particular for $m>n, K_{n} \cap T=\phi$. Let $K=\left(\bigcup_{n} K_{n}\right) \cup T$. Then $K$ is the desired set.

To show this, we first prove some lemmas.

Definition 5.1. The set

$$
V_{n}=\left\{\left(1-\frac{1}{n}, \frac{2 \pi k}{n}\right): 0 \leqq k<n\right\}
$$

will be called the set of vertices of $K_{n}$. Let $V=\bigcup_{n} V_{n}$. Then $V$ is called the set of vertices of $K$.

LEMMA 5.1. Let $h$ be a homeomorphism of $K$ onto itself such that, for some point $x_{0}$ in $T, h\left(x_{0}\right)=x_{0}$. Then $h \mid T$ is the identity.

(17) See $\$ 4$ for definition.

(18) J. de Groot in [8] and [9] has constructed continua with these properties, but from a somewhat different point of view. 
Proof. Let $\left\{v_{i}\right\}, v_{i} \in V_{i}$, be a sequence of vertices of $K$ converging to $x_{0}$. Then $h\left(v_{i}\right) \rightarrow x_{0}$ also. Since $h \mid K_{i}$ is a rotation, it follows that $\left\{h \mid K_{i}\right\}$ converges to a rotation of $T$, which must be the 0 -rotation (since $h\left(x_{0}\right)=x_{0}$ ); that is, $h \mid T$ must be the identity.

DEFINITION 5.2. Let $r$ be a rotation of Tonto itself. An extension $h$ of $r, h: K \rightarrow K$ is called a *-extension if $d(h, e)=d(r, e \mid T)$.

LeMma 5.2. Let $r$ be a rotation of $T$ onto itself with $r<\pi$. Then there exists $a^{*}$-extension $h$ of $r$.

Proof. Let $r_{n}$ be the counterclockwise period $n$ rotation about the origin in $E^{2}$. For each positive integer $n$, let $k_{n}$ be the largest nonnegative integer less than $n$, for which $r_{n}^{k_{n}}$ is a rotation which is less than or equal to $r$. Let $h: K \rightarrow K$ be defined by $h\left|K_{n}=r_{n}^{k_{n}} / K_{n} ; h\right| T=r$. Then $h$ is a *-extension of $r$.

LEMMA 5.3. Let $h$ be a homeomorphism of $K$ onto itself. Then $h \mid T$ is a rotation.

Proof. Suppose $h \mid T$ is not a rotation. Let $x_{0} \in T$ such that $h\left(x_{0}\right) \neq x_{0}$, and let $r<\pi$ be a (possibly clockwise) rotation of $T$ onto itself such that $r\left(x_{0}\right)=h^{-1}\left(x_{0}\right)$. By Lemma 5.2, there exists an extension $r^{\prime}$, of $r$, to $K$. Let $g=h r^{\prime}$. Then $g\left(x_{0}\right)=x_{0}$, for $g\left(x_{0}\right)=h\left(r^{\prime} x_{0}\right)=h\left(r^{\prime}\left(x_{0}\right)\right)=h\left(h^{-1}\left(x_{0}\right)\right)=x_{0}$. However, by Lemma 5.1, $g \mid T$ must be the identity; that is, $h \mid T=r^{-1}$, and therefore $h \mid T$ is a rotation. This is a contradiction.

LEMMA 5.4. $G(K)$ is complete.

Proof. Let $\left\{h_{i}\right\}$ be a Cauchy sequence of homeomorphisms on $K$. Since $K$ is compact, $\left\{h_{i}\right\}$ converges to a limit map, $h$. Since $K_{n}$ admits only finitely many homeomorphisms, there exists an integer, $I_{n}$, such that for $i, j>I_{n}, h_{i}\left|K_{n}=h_{j}\right| K_{n}$. Therefore, for each $n, h \mid K_{n}$ is a homeomorphism, and thus $h$ is 1-1 off $T$. Now, by Lemma $5.3, h_{n} \mid T$ is a rotation for each $n$. Since $\left\{h_{n}\right\}$ is Cauchy, we have a Cauchy sequence of rotations on $T$, and this must converge to a rotation. Therefore $h$ is $1-1$ on $T$. Since $h$ is $1-1$, and $K$ is compact, it follows that $h$ is a homeomorphism.

LEMMA 5.5. Let $W=\{h \in G(K): h \mid T$ is the identity $\}$. Let $h_{0}$ be any homeomorphism in $G(K)$ such that $h_{0}|T \neq e| T$, and let $W^{\prime}=\left\{h \in G(K): h\left|T=h_{0}\right| T\right\}$. Then $W$ and $W^{\prime}$ are homeomorphic.

Proof. Define a function, $\phi$, on $W$, by $\phi(h)=h h_{0}$. The proof that $\phi$ is a homeomorphism is fairly standard.

LEMMA 5.6. For each $\varepsilon>0$, there exists a $\delta, 0<\delta<\varepsilon$, such that if $h \in G(K)$ for which $d(h, e)=\delta$, then the only points of $K$ which move a distance $\delta$ under $h$ are the points of $T$. 
Proof. We first observe that since there are only a finite number of homeomorphisms on each $K_{n},\left\{h \mid K_{n}: h \in G(K)\right\}$ is finite; therefore there are only a finite number of distances between $h\left|K_{n}, e\right| K_{n}$. It follows that

$$
D=\left\{d: d=d\left(h\left|K_{n}, e\right| K_{n}\right) \text { for some } n\right\}
$$

is countable. Let $\delta<\varepsilon, \delta \notin D$, and let $h \in G(K)$ for which $d(h, e)=\delta$. By Lemma 5.2, we know such an $h$ exists.

We show that the only points of $K$ which move a distance $\delta$ under $h$ are the points of $T$. Since $d(h, e)=\delta$, no point moves more than a distance $\delta$ away from itself. Further, no point of any $K_{n}$ can move a distance $\delta$ away from itself, for then $d\left(h\left|K_{n}, e\right| K_{n}\right) \geqq \delta$. But $d(h, e)=\delta$ and $\delta \notin D$.

It follows that the only points which move a distance $\delta$ under $h$ are the points of $T$.

\section{TheOREM 5.1. $G(K)$ is at least 1-dimensional.}

Proof. Let $U$ be a neighborhood of the identity of diameter $<3 / 2$. We will obtain a sequence of pairs of homeomorphisms $\left\{h_{j}, g_{j}\right\}$ such that $h_{j} \in U, g_{j} \in C(U), d\left(h_{j}, g_{j}\right)<\pi / 2^{j-1}$, and $h_{j} \rightarrow h$, a homeomorphism in $G(K)$. Then $h \in \operatorname{Bd} U$.

In the following, $r_{j}$ denotes a rotation of $\pi / 2^{j}$ radians on $T$. Let $k_{1}$ be the smallest nonnegative integer such that $r_{1}^{k_{1}}$ has a ${ }^{*}$-extension $h_{1} \in U$, but for which any *-extension of $r_{1}^{k_{1}+1} \in C(U)$. Let $\psi_{1}$ be a *-extension of $r_{1}$, and let $g_{1}=\psi_{1} h_{1}$. We note that $g_{1}$ is a ${ }^{*}$-extension of $r_{1}^{k_{1}+1}$. Then $h_{1} \in U, g_{1} \in C(U)$, and $d\left(h_{1}, g_{1}\right)<\pi$.

We proceed by induction. Assume that we have $h_{j-1}, g_{j-1}$ such that $h_{j-1} \in U, g_{j-1} \in C(U), g_{j-1}=\psi_{j-1} h_{j-1}$ where $\psi_{j-1}$ is a ${ }^{*}$-extension of $r_{j-1}$, and $\psi h_{j-1} \in C(U)$ for any *-extension $\psi$ of $r_{j-1}$. Let $k_{j}$ be the smallest nonnegative integer for which there exists a ${ }^{*}$-extension $\phi_{j-1}$ of $r_{j}^{k_{j}}$ such that $\phi_{j-1} h_{j-1} \in U$, but any *-extension $\phi$ of $r_{j}^{k_{j}+1} \in C(U)$. Let $h_{j}=\phi_{j-1} h_{j-1}$, and let $g_{j}=\psi_{j} h_{j}$

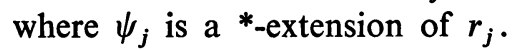

We now have a sequence of pairs of homeomorphisms $\left\{h_{j}, g_{j}\right\}$ such that $d\left(h_{j-1}, h_{j}\right)<\pi / 2^{j-2}$. Therefore $\left\{h_{j}\right\}$ is Cauchy, and by Lemma $5.4, h_{j} \rightarrow h$, where $h$ is a homeomorphism of $K$ onto itself. Since $d\left(h_{j}, g_{j}\right)<\pi / 2^{j-1}$, and $\pi / 2^{j-1} \rightarrow 0, g_{j} \rightarrow h$ also. Therefore $h \in \mathrm{Bd} U$. It follows that $G(K)$ is at least 1-dimensional.

LEMMA 5.7. Let $\varepsilon$ be a number such that $0<\varepsilon<1$ and $d\left(h\left|K_{n}, e\right| K_{n}\right) \neq \varepsilon$, for all $h, n$. Let $G_{\varepsilon}=\{h \in G(K): d(h, e)=\varepsilon\}$. Then $G_{\varepsilon}$ is 0-dimensional.

Proof. By Lemma 5.6, the hypothesis can be satisfied; it also follows that $G_{\varepsilon}$ is a subset of the set of homeomorphisms, $G_{\varepsilon}^{\prime}$, which are extensions of a rotation $T$ which moves points of $T$ a distance $\varepsilon$. By Lemma 5.5, $G_{\varepsilon}^{\prime}$ is homeomorphic to $W=\{h \in G(K): h \mid T$ is the identity $\}$. 
We show that $W$ is 0 -dimensional. It is sufficient to show that $W$ is 0 -dimensional at the identity, $e$. To this end, let $\left\{\varepsilon_{i}\right\}$ be a sequence of positive numbers, $\varepsilon_{i} \rightarrow 0$, such that if $h \in G(K)$, then $d\left(h\left|K_{n}, e\right| K_{n}\right) \neq \varepsilon_{i}$, for all $i, n$. Let $S_{\varepsilon_{i}}=\left\{h \in W: d(h, e) \leqq \varepsilon_{i}\right\}$. Bd $S_{\varepsilon_{i}}$ is a subset of $H=\left\{h \in W: d(h, e)=\varepsilon_{i}\right\}$. But $H=\phi$, since the only homeomorphisms which are a distance $\varepsilon_{i}$ from $e$, must move points on $T$, but $H$ is a set of extensions of the identity on $T$. Therefore $\operatorname{Bd} S_{\varepsilon_{i}}=\phi$. Thus we have a sequence of spheres about $e$, converging to $e$, and whose boundaries are empty. It follows that $W$, and therefore $G_{\varepsilon}$, is 0 -dimensional.

THEOREM 5.2. $G(K)$ is at most 1-dimensional.

Proof. Let $\left\{\varepsilon_{i}\right\}$ be a sequence of positive numbers such that $\varepsilon_{i} \rightarrow 0$ and $d\left(h\left|K_{n}, e\right| K_{n}\right) \neq \varepsilon_{i}$, for all $n, i$. Let $S_{\varepsilon}=\left\{h \in G(K): d(h, e) \leqq \varepsilon_{i}\right\}$. Then $\operatorname{Bd} S_{\varepsilon_{i}} \subset G_{\varepsilon_{i}}=\left\{h \in G(K): d(h, e)=\varepsilon_{i}\right\}$. By Lemma 5.7, $G_{\varepsilon_{i}}$ is 0-dimensional. Therefore $\mathrm{Bd} S_{\varepsilon}$ is 0-dimensional. It follows that $G(K)$ is at most 1-dimensional at the identity. Therefore $G(K)$ is at most 1-dimensional.

THEOREM 5.3. $G(K)$ is exactly 1-dimensional.

Proof. By Theorem 5.1, $\operatorname{dim} G(K) \geqq 1$, and by Theorem $5.2, \operatorname{dim} G(K) \leqq 1$. Therefore $\operatorname{dim} G(K)=1$.

REMARK 1. Since there are uncountably many different dendrons which could have been used in the construction, there are uncountably many such compact spaces.

REMARK 2. By using continua $K_{p^{n}}$, we obtain a set $K$ for which rotations of period $p^{n}$ on $T$ can be extended to rotations of period $p^{n}$ on $K$. However, if we use continua $K_{\alpha_{n}}$, where the sequence $\left\{\alpha_{n}\right\}$ includes an infinite sequence of relatively prime integers, then no rotation of $T$ of any finite positive period can be extended to a homeomorphism of $K$ of that period.

THEOREM 5.4. $G(K)$ is totally disconnected.

Proof $\left({ }^{19}\right)$. We show that any pair of homeomorphisms of $G(K)$ can be separated. Let $g_{1}, g_{2} \in G(K), g_{1} \neq g_{2}$. Then there exists an integer $n>0$, such that $g_{1}\left|K_{n} \neq g_{2}\right| K_{n}$. Let $A=\left\{h \in G(K): h\left|K_{n}=g_{1}\right| K_{n}\right\}$, and let $B=G(K)-A$. Then $g_{1} \in A, g_{2} \in B$, and $A \cup B=G(K)$. Let $h_{0}$ be any element of $A$, and let $g_{0}$ be any element of $B$. Then $h_{0}$ and $g_{0}$ differ on $K_{n}$ and therefore must be a positive distance apart - at least $d\left(e\left|K_{n}, r_{n}\right| K_{n}\right)$ where $r_{n}$ is the rotation of $2 \pi / n$ radians on $K_{n}$. Therefore $A$ and $B$ are mutually separate, and $G(K)$ is totally disconnected.

THEOREM 5.5. $G(K)$ is abelian.

(19) This proof is similar to the proof of Theorem 1.2, suggested by R. D. Anderson to show that the group of homeomorphisms of the universal plane curve is totally disconnected. 
Proof. Let $h, g \in G(K)$. Since $K_{n}$ goes onto itself under any homeomorphisr.. in $G(K)$, and $h$ and $g$ commute on $K_{n}$, as well as on $T$, it follows that $h g=g h$. Thus $G(K)$ is abelian.

Thus we have completed construction of the auxiliary space $K$. Our next objective is to construct a continuum $M$ such that $G(M)$ is topologically and algebraically the same as $G(K)$.

Construction of $M$. We will "extend" the set $K$. We first modify the set $K$ in the following way: Let $C=T \times[0,1]$ in $E^{3}$, where $T \subset x y$-plane and $[0,1]$ is on the $z$-axis. Now consider the vertical unit interval in $E^{3}$ above the point in $E^{2}$, the $z=0$ plane, with polar coordinates $(1-1 / n, 0)$. Put a dendron $D_{n}$, with a dense set of branch points of different orders greater than 3 , in a small neighborhood of this interval in such a way that

(1) $D_{n}$ contains this interval,

(2) $D_{n} \cap E^{2}$ is an endpoint of $D_{n}$,

(3) $D_{n}$ intersects the plane $z=1$ in an endpoint of $D_{n}$,

(4) $D_{n} \cap r_{n}^{i}\left(D_{n}\right)=\varnothing$, where $r_{n}$ is the period $n$ positive rotation about the $z$-axis in $E^{3}$, and $1 \leqq i<n$,

(5) $r_{n}^{i}\left(D_{n}\right) \cap r_{m}^{j}\left(D_{m}\right)=\varnothing$, for $n \neq m$, and all $i, j$ such that $0 \leqq i<n$ and $0 \leqq j<m$, and

(6) $D_{n} \cap C=\varnothing$, for all $n$.

Let $K_{n}^{\prime}=K_{n} \cup \bigcup_{i=0}^{n-1} r_{n}^{i}\left(D_{n}\right)$. Let $M^{\prime}=\left(\bigcup_{n} K_{n}^{\prime}\right) \cup C$. Let $h$ be a level preserving map of $E^{3}$ onto itself, which is

(1) the identity on $E^{2}$,

(2) a homeomorphism on each level other than $z=1$, and

(3) carries the unit disk of the plane $z=1$ to the point $(0,0,1)$.

Let $M=h\left(M^{\prime}\right)$. Then $M$ is a continuum with the desired properties.

THEOREM 5.6. There exists a continuum $M$ such that $G(M)$ is totally disconnected, abelian, and exactly 1-dimensional.

Proof. Clearly the continuum $M$ constructed above is a continuum in which every homeomorphism $g$ of $K$ onto itself can be extended to a homeomorphism $h$ of $M$ onto itself. Further, this extension is unique, and if $g_{i} \rightarrow g$, then the extensions $h_{i}$ of $g_{i}$ converge to the extension $h$ of $g$. Therefore $G(K)$ and $G(M)$ are topologically the same.

It is also clear from the construction that they are algebraically the same.

Thus it follows from Theorems 5.3, 5.4, and 5.5 that $G(M)$ is totally disconnected, abelian, and exactly 1-dimensional.

The next portion of this section is devoted to generalizing the results of Theorem 5.6 above. We wish to construct continua $M_{n}$ such that $G\left(M_{n}\right)$ is homeomorphic to a product of $n$ 1-dimensional groups. Again we begin by making some auxiliary constructions. 
Construction of the continua $P_{n, p_{1}, \ldots, p_{n}}$. For each positive integer $n$, and each $n$ relatively prime integers $p_{1}, \cdots, p_{n}, p_{i}>2$, we construct a continuum $P_{n, p_{1}, \ldots, p_{n}}$ such that $G\left(P_{n, p_{1}, \ldots, p_{n}}\right)$ is the product of $n$ cyclic groups of orders $p_{1}, \cdots, p_{n}$ respectively $\left({ }^{20}\right)$.

Let $T_{n}$ be the $n$-dimensional torus, thought of as the product of $n$ circles, $C_{1}, \cdots, C_{n}$; that is, $T_{n}=\prod_{i=1}^{n} C_{i}$. Let $r_{i}$ be a rotation of order $p_{i}$ on $C_{i}$, and let $h\left(r_{1}^{i_{1}}, r_{2}^{i_{2}}, \cdots, r_{n}^{i_{n}}\right)$ be a homeomorphism of $T_{n}$ onto itself which is the product of the rotations $r_{j}^{i_{j}}$ on $C_{j}$. Then $H_{n}=\left\{h\left(r_{1}^{i_{1}}, r_{2}^{i_{2}}, \cdots, r_{n}^{i_{n}}\right): 0 \leqq i_{j}<p_{j}\right\}$ is a set of homeomorphisms of $T_{n}$ onto itself which is the product of $n$ cyclic groups, $G_{i}$, of order $p_{i}$. We assume that we have a "rectangular" grid of $T_{n}$ in such a way that if $R$ is any fixed $n$-rectangle, then $\bigcup\left\{h(R): h \in H_{n}\right\}$ is $T_{n}$.

Now let $D_{1}$ be a dendron with a dense set of branch points of different orders constructed in a neighborhood of a 1-simplex of the 1-dimensional skeleton of the rectangular grid, in such a way that $h_{1}\left(D_{1}\right) \cap h_{2}\left(D_{1}\right)$ is either empty or a single point, if $h_{1}, h_{2} \in H_{n}, h_{1} \neq h_{2}$. Let $P_{n, p_{1}, \ldots, p_{n}}^{1}=\bigcup\left\{h\left(D_{1}\right): h \in H_{n}\right\}$.

Let $A_{2}$ be a 1-simplex in the 1-dimensional skeleton of the rectangular grid such that (1) $A_{2} \cap A_{1}$ is a single point and (2) $A_{2} \cap P_{n, 1}^{1}, \ldots, p_{n}$ consists of the two endpoints of $A_{2}$. Let $D_{2}$ be a dendron with a dense set of branch points of different orders, $D_{2}$ not homeomorphic to $D_{1}$, and constructed in a neighborhood of $A_{2}$ in such a way that

(1) $D_{2} \cap P_{n, p_{1}, \ldots, p_{n}}^{1}$ consists of the two endpoints of $A_{2}$, and

(2) $h_{1}\left(D_{2}\right) \cap h_{2}\left(D_{2}\right)$ is either empty or a single point, if $h_{1}, h_{2} \in H_{n}, h_{1} \neq h_{2}$.

Let $P_{n, p_{1}, \ldots, p_{n}}^{2}$ be $P_{n, p_{1}, \ldots, p_{.}}^{1} \cup \bigcup\left\{h\left(D_{2}\right): h \in H_{n}\right\}$.

We continue the above process inductively, each time choosing the arc $A_{i}$ so that $A_{i} \cap A_{1}$ is the same endpoint of $A_{1}$. After $n$ stages, we obtain the continuum, $P_{n}^{n}, p_{1}, \ldots, p .$. .

Let $P_{n, p_{1}, \ldots, p_{n}}=P_{n, p_{1}, \ldots, p_{n}}^{n}$. Then $P_{n, p_{1}, \ldots, p_{n}}$ satisfies the conditions of the first paragraph.

THEOREM 5.7. For each positive integer $n$, there exists a compact set $Q_{n}$ such that $G\left(Q_{n}\right)$ is homeomorphic to the product of $n$ 1-dimensional groups, and thus $1 \leqq \operatorname{dim} G\left(Q_{n}\right) \leqq n$.

Outline of the construction of $Q_{n}$. Let $\left\{p_{1, i}\right\},\left\{p_{2, i}\right\}, \cdots,\left\{p_{n, i}\right\}$ be $n$ increasing sequences of positive integers such that $\lim _{i \rightarrow \infty} p_{k, i}=\infty$, and such that, for each $i$, each pair $p_{k, i}$ and $p_{l, i}$ are relatively prime. Let $P_{n, p_{1}^{j}, \ldots, p^{j}}$ be a continuum as

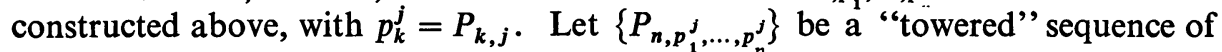
these sets converging (from the interior) to a limit $n$-torus, $T_{n}$. Then $Q_{n}=\left(\bigcup_{j}\left\{P_{n, p_{1}^{j}, \ldots, p_{-}^{j}}\right\} \cup T_{n}\right)$ is the compact set of the theorem, since $G\left(Q_{n}\right)$ will just be the product of $n$ 1-dimensional groups, each of which is obtained by considering the homeomorphisms which move points in "one direction" only. The proof is like that of Theorem 5.3.

(20) See footnote 18. 
NoTE. By a "towered" sequence of sets $\left\{S_{i}\right\}$, we mean that there exists a sequence of tori $\left\{T_{i}\right\}$ such that $S_{i} \subset T_{i}$ and $T_{i}$ is a subset of the "interior" of $T_{i+1}-$ i.e. the bounded complementary domain of $T_{i+1}$.

THEOREM 5.8. For each positive integer $n$, there exists a continuum $M_{n}$ such that $G\left(M_{n}\right)$ is homeomorphic to the product of $n$ 1-dimensional groups, and thus $1 \leqq \operatorname{dim} G\left(M_{n}\right) \leqq n$.

Outline of construction of $M_{n}$. The compact set $Q_{n}$ is a subset of $E^{n+1}$. We think of the join of the limit torus $T_{n}$ and the set of "vertices" $V$ of $Q_{n}$ with a point $q$ in $\left(E^{n+2}-\right.$ the $E^{n+1}$ hyperplane containing $\left.Q_{n}\right)$, and construct, in a neighborhood of each arc from $q$ to a vertex of $Q_{n}$, a dendron (containing this arc) with a dense set of branch points of different orders, much like we did for the continuum $M$ of Theorem 5.6, which had a 1-dimensional group. Then $G\left(M_{n}\right)$ is the same as $G\left(Q_{n}\right)$.

Our final objective of this section is to show, as promised in $\S 4$, that there are rational curves with 1-dimensional groups of homeomorphisms. Once again, we begin with some auxiliary constructions.

Outline of the construction of the continuum $Q$. We construct a continuum, $Q$, such that the group of all homeomorphisms of $Q$ is infinite cyclic $\left({ }^{21}\right)$. Consider the interval $I=[-1,1]$ on the $x$-axis in $E^{2}$ and the partition, $A$, of $I$ into an infinite number of subintervals by means of the points $1-1 / n$ and $-1+1 / n$, $n>1$, on $I$. Let $D$ be a dendron with a dense set of branch points of different orders, with $D$ constructed in a neighborhood of $\left[-\frac{1}{2}, \frac{1}{2}\right]$, containing this interval, and not meeting $[-1,1]$ elsewhere. We also require that $D$ meet each of the lines $x=-\frac{1}{2}$ and $x=\frac{1}{2}$, in exactly one point, an endpoint of $D$, and that the points of $D$ lie in the set of points of $E^{2}$ such that $-\frac{1}{2} \leqq x \leqq \frac{1}{2}$.

Let $h$ be any homeomorphism of $I \times\left[-\frac{1}{2}, \frac{1}{2}\right]$ onto itself carrying each of the subintervals of $I$, determined by $A$, onto the next subinterval, and carrying vertical lines onto vertical lines. Let

$$
Q=\overline{\bigcup_{i=-\infty}^{\infty} h_{i}(D) .}
$$

Clearly, $G(Q)$ is infinite cyclic.

THEOREM 5.9. There exists a rational curve $R$ such that $G(R)$ is exactly 1-dimensional.

Outline of construction of $R$ (and proof). Let $\left\{d_{1, i}\right\},\left\{d_{2, i}\right\}, \cdots,\left\{d_{n, i}\right\}, \cdots$ be an infinite sequence of increasing sequences of integers $>2$, such that $d_{i, j} \neq d_{k, l}$ whenever $i \neq k$.

Let $I_{j}$ be the interval from $(-1,1 / j)$ to $(1,1 / j)$ in $E^{2}$. Then $I_{j} \rightarrow I=[-1,1]$ on the $x$-axis.

(21) See footnote 18. 
Let $A_{j, 1}$ be the infinite partition of $I_{j}$ determined by the vertical lines $x=-1+1 / n$ and $x=1-1 / n$, for all $n>1$, as in the construction of $Q$ above. We refine $A_{j, 1}$ to obtain a partition, $A_{j, 2}$, by subdividing into halves each interval of $I_{j}$ determined by $A_{j, 1}$. We continue inductively, until we obtain the partition $A_{j, j}$.

Construct a continuum $Q_{j}$, on $I_{j}$, as in the construction of $Q$ above, by using a dendron $D_{j}$ whose branch points are of orders $d_{j, i}$ of the sequence $\left\{d_{j, i}\right\}$ above, and by using the partition $A_{j, j}$ for $I_{j}$. We further require that $Q_{i} \cap Q_{j}=\phi$ for $i \neq j$.

Let $R^{\prime}=\bigcup_{j} Q_{j} \cup I$.

Let $h$ be a map of $E^{2}$ onto itself carrying the vertical lines $x=\alpha$, for each real number $\alpha$, onto themselves, so that $h$ is a homeomorphism everywhere, except that $h([a, b])=(-1,0)$ and $h([c, d])=(1,0)$, where $[a, b]$ is the vertical interval from $(-1,0)$ to $(-1,1)$, and $[c, d]$ is the vertical interval from $(1,0)$ to $(1,1)$.

Let $R=h\left(R^{\prime}\right)$. Then clearly $R$ is a rational curve. The proof that $G(R)$ is 1-dimensional is similar to the proof that $G(M)$ (of Theorem 5.6) is 1-dimensional.

REMARK. We make the observation that if the partitions of the $I_{n}$ 's are chosen arbitrarily with only the provision that the mesh of the infinite partitions has limit 0 , it is possible to obtain a rational curve such that no homeomorphism of the limit arc onto itself, except the identity, can be extended, and therefore $G(R)$ would be 0 -dimensional.

COROLlary 5.9.1. For each positive integer $n$, there exists a rational curve $R_{n}$, such that $G\left(R_{n}\right)$ is homeomorphic to the product of $n$ 1-dimensional groups, and thus $1 \leqq \operatorname{dim} G\left(R_{n}\right) \leqq n$.

Outline of construction and proof. Let $R_{n}$ be the union of $n$ different (that is, nonhomeomorphic) continua, $R_{i}^{\prime}$, constructed like the continuum $R$ above, $R_{i}^{\prime}$ meeting $R_{i+1}^{\prime}$ at the right- and left-hand endpoints of their limit arcs respectively, $1 \leqq i<n$. Then any homeomorphism of $R_{n}$ onto itself carries each) $R_{i}^{\prime}$ onto itself, and the homeomorphisms on the different $R_{i}^{\prime}$ s are independent of each other. Therefore $G\left(R_{n}\right)$ is the same as $\prod_{i=1}^{n} G\left(R_{i}^{\prime}\right)$. Since each $G\left(R_{i}^{\prime}\right)$ is exactly 1-dimensional, and since the product of $n$ 1-dimensional sets is at most $n$-dimensional, $1 \leqq \operatorname{dim} G\left(R_{n}\right) \leqq n$.

Remark. We make the following observations (without proof) about the continua $M_{n}$ and their groups of homeomorphisms:

(1) There are uncountably many such continua.

(2) Every homeomorphism, $r$, which is a product of rotations on the limit torus $T_{n}$, can be extended to a homeomorphism, $h$, of $M_{n}$, onto itself, such that $d\left(r, e \mid T_{n}\right)=d(h, e)$.

(3) If $h \in G\left(M_{n}\right)$, then $h \mid T_{n}$ is a product of rotations. 
(4) Let $r$ be a product of rotations on $T_{n}$, let $A=\left\{h \in G\left(M_{n}\right): h \mid T_{n}=r\right\}$, and let $B=\left\{h \in G\left(M_{n}\right): h \mid T_{n}\right.$ is the identity $\}$. Then $A$ and $B$ are homeomorphic.

(5) Let $x_{0} \in T_{n}$, and let $\phi: G\left(M_{n}\right) \longrightarrow T_{n}$ be defined by $\phi(g)=g\left(x_{0}\right)$. Then $\phi$ is a continuous, open, homomorphism of $G\left(M_{n}\right)$ onto the $n$-dimensional torus.

QUESTIONS. In view of the theorems of this section, and the statements above, we raise the following questions:

(1) Is $\operatorname{dim} G\left(M_{n}\right)=n$ ?

(2) Is $\operatorname{dim} G\left(R_{n}\right)=n$ ? ( $R_{n}$ is the curve of Corollary 5.9.1).

REMARK. We note that if the answer to Question (1) is "no", then we have examples of continuous, open, dimension-raising homomorphisms from the groups of homeomorphisms of continua onto compact groups.

\section{BIBLIOGRA PHY}

1. R. D. Anderson, The group of all homeomorphisms of the universal curve, Abstract 323, Bull. Amer. Math. Soc. 63 (1957), p. 143.

2. - One-dimensional continuous curves and a homogeneity theorem, Ann. of Math. 68 (1958), 1-16.

3. G. T. Whyburn, Topological characterization of the Sierpinski curve, Fund. Math. 45 (1958), 320-324.

4. —, Analytic topology, Amer. Math. Soc. Colloq. Publ. Vol. 28, Amer. Math. Soc. Providence, R. I., 1942; reprint 1955.

5. Karl Menger, Kurventheorie, Teubner, Leipzig and Berlin, 1932.

6. W. Hurewicz and H. Wallman, Dimension theory, Princeton Univ. Press, Princeton, N. J., 1948.

7. R. L. Moore, Foundations of point set theory, Amer. Math. Soc. Colloq. Publ. Vol. 13, Amer. Math. Soc., Providence, R. I., 1932; revised 1962.

8. J. de Groot and R. J. Wille, Rigid continua and topological group-pictures, Arch. Math. 9 (1958), 441-446.

9. J. de Groot, Groups represented by homeomorphism groups. I, Math. Ann. 138 (1959), 80-102.

\section{Louisiana State University in New Orleans, NeW ORLEans, Louisiana}

